\title{
Title: Development and validation of serological markers for detecting recent exposure to Plasmodium vivax infection
}

Authors: Rhea J. Longley ${ }^{1,2,3} \dagger$, Michael T. White ${ }^{4} \dagger$, Eizo Takashima ${ }^{5}$, Jessica Brewster ${ }^{1}$, Masayuki Morita $^{5}$, Matthias Harbers ${ }^{6,18}$, Leanne J. Robinson ${ }^{1,2,7}$, Fumie Matsuura ${ }^{6}$, Shih-Jung (Zoe) Liu ${ }^{1,2}$, Connie S. N. Li-Wai-Suen ${ }^{1,2}$, Wai-Hong Tham ${ }^{2,8}$, Julie Healer ${ }^{2,8}$, Christele Huon ${ }^{9}$, Chetan E. Chitnis 9 , Wang Nguitragool ${ }^{10}$, Wuelton Monteiro ${ }^{11,12}$, Carla Proietti ${ }^{13,14}$, Denise L. Doolan $^{13,14}$, Xavier C. Ding ${ }^{15}$, Iveth J. Gonzalez ${ }^{15}$, James Kazura ${ }^{16}$, Marcus Lacerda ${ }^{11,17}$, Jetsumon Sattabongkot ${ }^{3}$, Takafumi Tsuboi ${ }^{5}$, Ivo Mueller ${ }^{1,2,4} *$

\section{Affiliations:}

${ }^{1}$ Population Health and Immunity Division, Walter and Eliza Hall Institute of Medical Research, Melbourne, Australia.

${ }^{2}$ Department of Medical Biology, University of Melbourne, Melbourne, Australia.

${ }^{3}$ Mahidol Vivax Research Unit, Faculty of Tropical Medicine, Mahidol University, Bangkok, Thailand.

${ }^{4}$ Malaria Parasites \& Hosts Unit, Department of Parasites \& Insect Vectors, Institut Pasteur, Paris, France.

${ }^{5}$ Division of Malaria Research, Proteo-Science Center, Ehime University, Matsuyama, Japan.

${ }^{6}$ CellFree Sciences Co., Ltd., Yokohama, Japan.

${ }^{7}$ Burnet Institute, Melbourne, Australia.

${ }^{8}$ Infection and Immunity Division, Walter and Eliza Hall Institute of Medical Research, Melbourne, Australia

${ }^{9}$ Malaria Parasite Biology and Vaccines, Department of Parasites \& Insect Vectors, Institut Pasteur, Paris, France.

${ }^{10}$ Department of Molecular Tropical Medicine and Genetics, Faculty of Tropical Medicine, Mahidol University, Bangkok, Thailand.

${ }^{11}$ Fundacão de Medicina Tropical Dr. Heitor Vieira Dourado, Manaus, Brazil.

${ }^{12}$ Universidade do Estado do Amazonas, Manaus, Brazil.

${ }^{13}$ Centre for Molecular Therapeutics, Australian Institute of Tropical Health and Medicine, James Cook University, Cairns, Australia.

${ }^{14}$ QIMR Berghofer Medical Research Institute, Brisbane, Australia.

${ }^{15}$ Foundation for Innovative New Diagnostics, Geneva, Switzerland.

${ }^{16}$ Center for Global Health and Diseases, Case Western Reserve University, Cleveland, United States.

${ }^{17}$ Instituto Leônicas \& Maria Deane (Fiocruz), Manaus, Brazil.

${ }^{18}$ RIKEN Center for Integrated Medical Sciences (IMS), Yokohama, Japan. 
*To whom correspondence should be addressed: mueller@wehi.edu.au.

$\uparrow$ These authors contributed equally.

One Sentence Summary: The manuscript describes identification and validation of a novel panel of $P$. vivax proteins that can be used to detect recent exposure to $P$. vivax infections within the prior 9 months.

Keywords: malaria, Plasmodium vivax, serological markers, exposure, surveillance, naturally acquired immunity, hypnozoites, malaria elimination, RBP2b, RAMA, MSP1, MSP3, MSP8, EBP, Pv-fam-a

Abstract: In order to accelerate towards malaria elimination, improved targeting of limited resources is essential. A major gap in our elimination toolkit for Plasmodium vivax malaria is the identification of individuals carrying arrested liver stages, called hypnozoites. These clinically silent but frequently relapsing hypnozoites are key to $P$. vivax persistence. Whilst hypnozoites cannot be directly detected, individuals who have had recent exposure to $P$. vivax and have not been treated are likely to harbor these parasites. By measuring IgG antibody responses to over $300 P$. vivax proteins, a panel of serological markers capable of detecting exposure to $P$. vivax infections in the prior 9-month period was identified and validated. Using antibody responses to $8 P$. vivax proteins, $80 \%$ sensitivity and specificity for detecting recent infections were achieved in three independent studies conducted in Thailand, Brazil and the Solomon Islands. As these individuals have a high likelihood of harboring hypnozoites, the suite of these 8 antibody responses can serve as biomarkers for the identification of individuals who should be targeted for treatment with liver-stage drugs such as primaquine and tafenoquine in mass drug administration programs aimed at controlling and eliminating $P$. vivax malaria.

\section{[Main Text: ]}

\section{Introduction}

Elimination of malaria by the year 2030 is now the explicit goal of all malaria endemic countries in the Asia-Pacific. While impressive progress towards this goal has been made, global funding for malaria control has flat-lined since 2010 and progress has stalled in many parts of the world (1). New interventions and tools for better targeting of limited resources are urgently needed.

A major hurdle for elimination is the increasing proportion of malaria cases caused by Plasmodium vivax as transmission declines $(1,2)$. P. vivax has a number of unique biological features that make its control difficult (3), including very high prevalence of low density, asymptomatic infections (4) and an arrested liver-stage that can reactivate weeks to months later to cause relapsing infections that cause morbidity as well as sustain transmission. These hypnozoites, which are not detectable by any current diagnostic method, can be responsible for over $80 \%$ of all blood-stage infections (5). Identifying and targeting individuals with hypnozoites is thus essential for accelerating and achieving malaria elimination. In addition, as endemicity decreases, malaria transmission becomes increasingly fragmented and highly seasonal $(6,7)$ rendering blanket approaches to malaria control and elimination inefficient. This requires new, 
innovative tools and approaches specifically designed to assist in targeting interventions to the changing malaria epidemiological landscape (8).

National malaria control programs still rely almost exclusively on microscopy or rapid diagnostic tests for the routine detection of malaria cases at health clinics and for surveillance using mass blood surveys or reactive case-detection (RACD) (9). While relatively cheap and readily available, these tools have limited sensitivity for detecting individuals with asymptomatic malaria who have lower parasite densities (10), making it difficult for control programs to efficiently identify and delineate areas of low and high $P$. vivax transmission and target their resources accordingly. Molecular techniques such as PCR have much greater sensitivity (10) but are rarely implemented by control programs due to high cost and the need for a specialised laboratory. All of these methods can only detect individuals with a current blood-stage infection, rendering mass screening and treatment (MSAT) approaches ineffective for reducing $P$. vivax transmission because they fail to treat individuals that only carry hidden $P$. vivax liver-stage infections $(5,11)$ with no circulating blood-stage parasitemia. Mass drug administration (MDA) is predicted to be highly effective as a control tool but only if it also includes a drug that targets $P$. vivax hypnozoites (5). Unfortunately, primaquine and tafenoquine, the only current drugs able to eliminate hypnozoites, can have toxic side effects in glucose-6-phosphate dehydrogenase (G6PD) deficient individuals (12), limiting their acceptability for MDA campaigns without prior G6PD screening, in particular in low transmission areas where $>90 \%$ MDA recipients will have no direct benefit from the treatment. There is thus a diagnostic gap, with MSAT ineffective due to under treatment and MDA unacceptable due to overtreatment. A strategy that can efficiently target individuals with current blood-stage infections and those with hypnozoites is required.

Blood-stage $P$. vivax infections induce a robust antibody response in humans to a broad range of $P$. vivax proteins, even following low-density asymptomatic infections (13). IgG antibody responses to specific Plasmodium proteins can be long-lived even after clearance of blood-stage infection $(14,15)$, and hence antibodies are markers of past exposure as well as current infections (15). Population-level serological exposure markers (SEM) have been used for surveillance of malaria and a number of other infectious diseases (i.e. leishmaniasis, influenza, trachoma), and antibody responses can be simply and cheaply measured in point-of-care tests (16-18). Besides general surveillance, SEMs may also be used for risk stratification and as guidance for targeted malaria control and elimination interventions (19). For $P$. vivax there is an important additional, individual-level application for SEMs: to target treatment to people at-risk of carrying clinically silent hypnozoites. While it is not possible to directly detect hypnozoites with current technology, all tropical and sub-tropical $P$. vivax strains cause a primary infection followed by a first relapse after no more than 6-9 months (20-22). Therefore, any individual with blood-stage infection within the previous 9 months, who has not received anti-liver-stage drugs, is likely to be a hypnozoite carrier. A carefully selected panel of proteins inducing antibodies that signify exposure within the previous 9 months could provide an accurate measure of current transmission and also highlight individuals likely to harbour hypnozoites who could be targeted for treatment with liver-stage drugs, such as primaquine or tafenoquine, after serological testing ("serological testing and treatment", seroTAT).

$342 P$. vivax proteins in this study, identified from the $P$. vivax genome (23), were screened for their ability to detect recent exposure to the parasite, and their use was subsequently validated in malaria-endemic regions of Thailand, Brazil and the Solomon Islands. 


\section{Results}

Antigen discovery phase

Data on the magnitude and longevity of IgG responses to $342 P$. vivax proteins following symptomatic $P$. vivax infections (24) were used to identify suitable proteins for detecting recent exposure (Fig. 1a). IgG was measured in four longitudinal plasma samples from individuals in Thailand $(n=32)$ and Brazil $(n=33)$ to enable estimation of antibody half-life (24) (Table S1).

A down-selection pipeline was developed to identify candidate serological markers using this data (Fig. 2). P. vivax proteins were first prioritised, with selection of those that had similar estimated IgG half-lives in both antigen discovery cohorts (Thailand and Brazil), and those that were highly immunogenic at the time of infection (at least $50 \%$ seropositive individuals) (Fig. 2a). Using these, 142 of the $342 P$. vivax proteins were prioritised as having suitable characteristics for use as candidate SEMs.

A statistical model for estimating time since infection was used to test the ability of the 142 prioritised proteins, individually or in combination, to predict time since last infection. Fig. 3a,b shows an example of dynamics of antibody responses to five proteins in two representative individuals from Thailand and Brazil. Fig. 3c,d shows the estimated time since last infection with uncertainty for antibody responses measured to the 5 proteins in Fig. 3a,b at 6 months after infection, for the same two representative individuals. Increasing the number of proteins to at least five resulted in higher accuracy compared to using a single protein alone, with diminishing returns beyond 20 proteins (Fig. S4). A simulated annealing algorithm was employed to determine optimal combinations of proteins that maximise the likelihood of correctly estimating the time since last infection (Fig. 3e). Whilst some proteins had 100\% probability of being included in a successful panel of a set size (Fig. 3e), there was some redundancy in choice of additional proteins and hence all were ranked based on their probability of inclusion in a 20protein panel (given the diminishing returns beyond 20 proteins). The 24 highest ranked proteins were selected for further testing.

The wheat germ cell free (WGCF) system was selected for expression of the proteins due to its eukaryotic nature and high rates of past success in expressing Plasmodium proteins (25). Despite all proteins being expressed in this system previously as crude proteins (24) not all could be produced at high yield and high purity, and thus an additional round of down-selection using ranking from the simulated annealing algorithm was performed to increase the number of proteins retained at this point in the pipeline. The algorithm was run on 104 of the 142 prioritised proteins, excluding the top 24 already selected (to provide more discriminatory power for selecting additional proteins) and 14 proteins known to have low yields or aggregates from our previous work. An additional 31 proteins were selected in the second round using the same methodology, with 55 P. vivax proteins in total identified as suitable candidate SEMs. Of these, 40 were successfully produced at a high yield and purity using the WGCF system (Table 2, Table S2).

An additional 20 proteins that were known to be highly immunogenic were added to these 40 proteins (26-29). This included four proteins already within the down-selected panel of 40 (different constructs or produced by a different laboratory and protein expression systems), and three distinct constructs of PVX_110810 (Duffy binding protein, each representing a different region of the protein or sequence from a different strain) (Table 2, Table S2). 


\section{Validation phase}

The next step in the down-selection pipeline was to validate the use of the 60 candidate SEMs in larger geographically diverse cohorts with a known history of malaria infections in the preceding 12 months, using plasma samples from three, one-year long observational cohort studies conducted in Thailand, Brazil and the Solomon Islands (Fig. 1b, Table 1). Individuals in these cohorts were assessed monthly for the presence of Plasmodium spp. by qPCR, with continuous concurrent passive case detection at local malaria clinics and hospitals. Plasma samples from the last visit of these cohorts $(n=829,928$ and 754 in Thailand, Brazil and the Solomon Islands, respectively) were used to measure IgG responses in a multiplex Luminex ${ }^{\circledR}$ assay to the $60 P$. vivax proteins. 158/2511 individuals in these cohorts had a concurrent $P$. vivax infection, detected by qPCR, at the time the plasma was collected; IgG antibody levels were strongly associated with current infection status for each of the 60 proteins in the Thai cohort (OR 2.1$7 \cdot 7, \mathrm{p}<0 \cdot 05)$ and for the majority of proteins in the Brazilian cohort $(57 / 60$, OR $1 \cdot 5-7 \cdot 1, \mathrm{p}<0 \cdot 05)$ (Table S3). This association was not as clear for the paediatric Solomon Islands cohort, with IgG levels to only 29 out of 60 proteins significantly associated with current $P$. vivax infections (OR $1 \cdot 7-6 \cdot 3, p<0 \cdot 05)$. Overall, there was a pattern of decreasing IgG magnitude with increasing time since last $P$. vivax infection (Fig. 4a-h, Fig. S5), with minimal reactivity in malaria naïve negative control individuals from Bangkok and Melbourne.

Recent infections were defined as PCR-confirmed infections occurring within the past 9 months. Using such a timeframe allows detection of likely hypnozoite carriers based on known relapse patterns (20), and enables analysis of individuals infected both before and after this period with the available data from the one-year long cohorts. Measurements of several individual proteins were able to classify individuals as infected with $P$. vivax within the past 9 months or not, with differing degrees of accuracy (Fig. 4i). Protein PVX_094255 (reticulocyte binding protein 2b, RBP $2 b$ ) reached $74 \%$ sensitivity and $74 \%$ specificity when used alone. IgG levels to the 60 candidate SEMs were correlated (Fig. 4j) with different distributions evident between the three geographic regions (Fig. 4k). A linear discriminant analysis (LDA) classification algorithm was used to identify combinations of the 60 candidate SEMs that could accurately predict recent infections. A degree of redundancy was found with multiple combinations of $P$. vivax proteins able to accurately predict recent infection when included in panels of up to eight proteins (Fig. S6). The top eight most frequently identified proteins when used in combination were: PVX_094255 (RBP2b (30)), PVX_087885 (rhoptry associated membrane antigen, RAMA (28), putative), PVX_099980 (merozoite surface protein 1, MSP1 (31)), PVX_096995 (tryptophanrich antigen (Pv-fam-a), PvTRAg_2 (32)), PVX_097625 (MSP8 (33), putative), PVX_112670 (unspecified product), KMZ83376.1 (erythrocyte binding protein II, EBPII (34)), and PVX_097720 (MSP3.10 (35)) (see Fig. 4i for ROC curves). PVX_112670 has previously been annotated as a tryptophan-rich antigen (PvTRAg_28 (32)). Table $\overline{2}$ and Table S2 show the individual ranking of all 60 proteins by $\mathrm{AUC}$, note that it is not necessarily the proteins with the highest individual AUC that work best in combination.

As shown in Table 2, for the top protein PVX_094255 (RBP2b), there were two protein constructs, mapping to different regions of the protein (RBP2 $\mathrm{b}_{161-1454}$ and RBP2 $\mathrm{b}_{1986-2653}$ ), produced in two separate laboratories using different expression and purification methods (30, 36). Antibody levels to the two constructs of PVX_094255 (RBP2 $\mathrm{b}_{161-1454}$ and RBP2 $\mathrm{b}_{1986-2653}$ ) were highly correlated (spearman $\mathrm{r}=0 \cdot 72, \mathrm{p}<0.0001$, all cohorts combined) and for this reason the construct that provided lower levels of classification accuracy (region encompassing aa1986- 
2653) was excluded from the top eight in order to increase the information content provided. RBP2 $b_{161-1454}$ induced very low levels of antibody reactivity in the malaria naïve control panels. When levels of background reactivity detected in the malaria naïve negative control panels were investigated in the other candidate SEMs, a significant negative association was observed between the mean antibody levels detected in the malaria naïve negative control panels $(n=274$ individuals, see Methods) and the ranking of the 60 SEMs determined from their individual receiver operating characteristic (ROC) curves (spearman $\mathrm{r}=0.6, \mathrm{p}<0.0001$, Fig. S10).

\section{Classification performance of an eight-protein SEM panel}

Fig. 5a-d presents ROC curves for assessing the classification performance of the top panel of eight SEMs for identifying individuals with exposure to $P$. vivax within the prior 9 months. There are diminishing returns in classification performance as the number of proteins is increased, with a plateau of $80 \%$ sensitivity and $80 \%$ specificity reached in all three geographic regions with five proteins. The performance of the classification algorithm across the different sample types was assessed. The algorithm correctly classifies more than $98 \%$ of malaria naïve negative controls, but classification performance was poor for individuals who had their last blood-stage $P$. vivax infection between $9-12$ months ago, with $30-60 \%$ of samples mis-classified (Fig. 6). Incorporating an individual's age into the classification algorithm did not result in substantial increases in classification accuracy (Fig. S11). There was a significant relationship between background levels of antibody in malaria naïve controls and selection of top proteins in the validation phase (Fig. S10). However, removing data from these malaria naïve control participants did not cause any substantial reductions in classification accuracy for the top eight proteins (Fig. S12). The cohort studies were conducted in regions co-endemic for $P$. vivax and $P$. falciparum, although the total number of individuals experiencing a $P$. falciparum infection during the study period was low ( $\mathrm{n}=19,31,22$ for Thailand, Brazil and the Solomon Islands, respectively). No associations were observed between recent $P$. falciparum infections and antibody level to the top eight proteins (Fig. S13).

The performance of targeted treatment conducted with the top eight $P$. vivax SEMs using a seroTAT approach compared to both MDA and MSAT with PCR was modelled (Fig. 5). Both sensitivity and specificity were assessed. In an MDA campaign, all hypnozoite carriers are targeted (except those deemed ineligible due to G6PD deficiency), but $>80 \%$ of the population receive unnecessary primaquine. In contrast for MSAT with PCR, no individual was over-treated (it is assumed that an individual with blood-stage $P$. vivax is a likely hypnozoite carrier). However, only $20 \%-40 \%$ of likely hypnozoite carriers are targeted. These approaches were compared to seroTAT with the panel of top eight SEMs at $80 \%$ sensitivity and specificity (Fig. 5). Across the three cohorts (Thailand, Brazil, Solomon Islands), at least $80 \%$ of individuals with hypnozoites are targeted (outperforming MSAT with PCR), with $20 \%$ or less of the population treated unnecessarily (substantially less than an MDA approach). Given the balance between sensitivity and specificity, two alternate targets were also modelled: $50 \%$ sensitivity and $98 \%$ specificity, and $98 \%$ sensitivity and $50 \%$ specificity. The high specificity approach results in no overtreatment but only slightly outperforms MSAT in terms of identifying hypnozoite carriers (45\% missed), and is likely not feasible for seroTAT. The high sensitivity approach identifies nearly all likely hypnozoites carriers and reduces overtreatment to approximately $50 \%$, compared to $>80 \%$ with MDA.

\section{Discussion}


There is a clear and urgent need for new tools and strategies if we are to achieve malaria elimination in the Asia-Pacific by the year 2030 (37). P. vivax, the predominant Plasmodium species in the Asia-Pacific, presents a unique challenge to elimination due to the presence of undetectable hypnozoites, representing a hidden burden of infection that contributes to maintaining residual transmission. A novel panel of candidate serological exposure markers ("SEMs") were identified and validated that allow for the detection of recent exposure to $P$. vivax within the prior 9-month period. Relapses from $P$. vivax infections are expected to occur at a frequency of every 1-2 months, with hypnozoites remaining in the liver for up to 1-2 years (21, 22). Although a few relapses may occur more than 1 year after the last blood-stage infection, the initial dormancy period for all $P$. vivax strains is less than 9 months with subsequent relapses occurring generally at shorter intervals (20-22). Apart from temperate 'hibernans' strains that are now restricted to the Korean Peninsula and have no primary infections, virtually all individuals who carry hypnozoites will thus have had a $P$. vivax blood-stage infection within the prior 9 months. To our knowledge, these SEMs represent the foundation for the first test that can indirectly identify likely hypnozoites carriers that could be targeted for treatment with liver-stage drugs.

We undertook an unbiased approach to choosing the best markers by starting the screen with a large panel of $342 P$. vivax proteins, and strategically selecting those that can predict recent infection history based on immunogenicity and antibody half-lives. The final panel, which has a sensitivity and specificity of $80 \%$ at identifying individuals with PCR-detectable blood-stage infection in the last 9 months in three geographically distinct regions, incorporates antibody responses to eight $P$. vivax proteins: PVX_094255 (RBP2b 161-1454), PVX_087885 (RAMA, putative), PVX_099980 (MSP1), PVX_096995 (tryptophan-rich antigen (Pv-fam-a)), PVX_097625 (MSP8, putative), PVX_112670 (unspecified product), KMZ83376.1 (EBPII), and PVX_097720 (MSP3.10). Most of these P. vivax proteins are not well described in the literature and only MSP1 and RAMA have previously been used, or suggested as, markers of exposure $(28,38-40)$. This highlights the success of our strategy in identifying novel exposure markers.

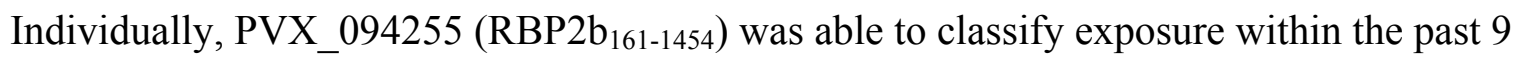
months with $74 \%$ sensitivity and $74 \%$ specificity. This may be sufficient for sero-surveillance and community-level targeting of preventative measures such as intensified vector control or focal test and treat (19). In such a scenario, lower sensitivity could be accounted for by increasing the sample size surveyed. Greater classification accuracy will be required for individual-level targeting for treatment with liver-stage drugs, for example in an elimination campaign using "serological testing and treatment" (seroTAT), where the goal is to treat all individuals who have hypnozoites or blood-stage P. vivax parasites. Combining PVX_094255 $\left(\mathrm{RBP} 2 \mathrm{~b}_{161-1454}\right)$ with additional proteins resulted in improved classification performance, albeit with diminishing returns. The final panel of eight proteins obtained $80 \%$ sensitivity and $80 \%$ specificity across the three geographic regions tested.

Anti-malarial antibody responses are affected by a number of factors, most notably time since last infection, intensity of infection, and age. Antibody responses may also be associated with other factors such as genetic variation in the human host or parasite that are challenging to account for. When considering the relationship between time since last infection and measured antibody responses, key properties of the data are the high level of variation (some individuals with blood-stage infection had very low responses, and some negative controls had very high responses), and the high degree of correlation. The analytic pipeline was designed to account for 
this type of data, and the possibility that recent infections may best be identified by combinations of antibody responses. However, rather than past infections being identified by complex antibody signatures and sophisticated algorithms, a number of simple factors contributed to good classification performance: (i) P. vivax proteins that can identify recent infections when used individually also do well in combinations; (ii) there are diminishing returns in classification performance as new $P$. vivax proteins are added to combinations; (iii) there are diminishing returns to algorithmic complexity: simple algorithms such as logistic regression capture most of the signal in the data, with more complex classifiers such as random forests providing incremental improvements in performance; (iv) there is no single best combination of antigens, rather many antigens are interchangeable; (v) there was no substantial advantage to including an individual's age once three or more antigens were included; and (vi) algorithms and combinations of antigens that provided good classification in one region also performed well in another region.

Although the performance of the pilot marker panel may seem low, several factors need to be taken into account when evaluating the potential performance of the SEMs. Firstly, given that individual episodes of $P$. vivax blood-stage can be relatively short (i.e. $<4-6$ weeks) (41), it is highly likely that some blood-stage infections may have been missed by qPCR in the cohorts used for validation of the SEMs as they had monthly active follow-up. In the analyses, such cases would be classified as false positive by the SEMs, when in reality the SEMs would have accurately detected these infections. Therefore, the real specificity of the test may well be higher. Similarly, a number of people (5-20\%) with PCR-positive infections at the time of antibody measurement were missed. This may represent individuals with very recent infections who have not yet generated a significant rise in antibody titres (42). These missed, concurrent infections could however be detected using an ultra-sensitive antigen capture test. A combination of antibody detection and antigen-capture assays - implemented either on a single diagnostic platform or two tests run in parallel - would therefore further increase the sensitivity of the diagnostic approach. Lastly, the SEMs are designed for application in low-transmission malariaendemic regions progressing towards elimination. Our data has previously shown that antibody responses are longer lasting in areas with higher transmission (24). In elimination contexts, overall (population) levels of immune responses to malarial antigens are low $(24,43)$, potentially increasing the difference between recently exposed and non-recently exposed individuals.

Although we anticipate optimal classification performance in near-elimination settings, our validation studies had to be conducted in low to moderate transmission settings, where the required sample sizes are operationally feasible. Indeed, in the three settings tested, classification performance was greatest in Thailand, the region with lowest transmission. The association of antibody levels to the 60 validation proteins with current $P$. vivax infections was less clear in the Solomon Islands compared to Thailand and Brazil, and the magnitude of the antibody response to these proteins was also generally lower. In contrast to the Thai and Brazilian cohorts that included individuals of all ages, the Solomon Islands study was a paediatric cohort. Antibody levels tend to increase with age in malaria-endemic regions $(13,29)$, and so the lower responses in the Solomon Islands may be due to the design of the cohort, which includes only children. However, the difference in antibody responses measured could also be influenced by other factors such as genetic diversity of $P$. vivax parasite proteins. Importantly, despite the lower magnitude antibody responses, the top eight SEMs could still accurately classify recent infections in the Solomon Islands. 
To further improve classification performance using the top $P$. vivax proteins identified, modifications of the protein constructs resulting in reduced background in malaria naïve control samples would be advantageous. The data demonstrated that proteins with lower levels of antibody reactivity in these controls performed better at classifying recent exposure. Addition of further purification steps, testing of other protein expression systems, design of smaller protein fragments (for those currently full-length), investigations of antigenic diversity and strainspecificity of antibody responses, could all be considered. Further information could potentially be gained by looking at antibody subclasses and/or IgM in addition to total IgG responses. Whilst antibody subclass responses will likely correlate with total $\mathrm{IgG}$, at least for the dominant subclass (44), the relationship with IgM is expected to be weaker (44) and acquisition and breadth of responses to malaria proteins has been shown to differ for IgG and IgM (45). Such optimisation and improvement of our assay may allow selection of a smaller panel of three to five proteins that will be able to reach the classification accuracy of our current panel of eight proteins. This would reduce the cost of running our SEMs in their current format (Luminex ${ }^{\circledR}$ assay) and also make a simpler point-of-care test feasible.

The SEMs current level of performance and their application in potential public health interventions such as seroTAT also needs to be seen in the context of the alternatives. MDA is a blanket approach, with liver-stage drugs given to all individuals within a defined region. Whilst this will effectively target all hypnozoite carriers and is predicted to achieve a high impact (but only if it includes an anti-hypnozoite drug (5)), it results in substantial overtreatment. In low transmission settings, up to $90 \%$ of people treated do not carry hypnozoites (i.e. the specificity of MDA can be as low at 6.5-23.3\%, Fig. 5) and do not derive any direct benefit from a treatment that can have negative consequences in G6PD-deficient individuals. Therefore, few P. vivax endemic countries are considering MDA with primaquine. MSAT interventions on the other hand will miss most hypnozoite carriers and when implemented with light microscopy or RDT diagnosis will not achieve any impact on $P$. vivax transmission (11). Even with a highly sensitive molecular test, MSAT is likely to miss $64.9-80.5 \%$ of the people carrying hypnozoites (Fig. 5) with relapsing infections thus predicted to rapidly erode its effect (5). Testing and treatment with SEMs thus result in less overtreatment compared to MDA, whilst still targeting a higher proportion of likely hypnozoites carriers compared to MSAT by PCR.

Similar efforts to develop SEMs are currently underway for $P$. falciparum (15). Whilst $P$. falciparum does not have a latent liver-stage leading to relapse, detecting recent exposure may still provide a useful tool for prioritising the limited resources available to areas with residual transmission. As numerous countries are endemic for both $P$. falciparum and $P$. vivax (1), efforts to develop SEMs for both species should be coordinated where possible. In addition, there is some evidence of antibody cross-reactivity between $P$. falciparum and $P$. vivax: cross-reactivity for $P$. falciparum and $P$. vivax MSP5 was demonstrated by a competitive antibody assay in PNG (co-endemic for $P$. falciparum and $P$. vivax) (46). Five of the top eight proteins have $P$. falciparum orthologs (with protein sequence identity ranging from 20.7-37.4\%) and thus need to be assessed for the impact of cross-reactivity in co-endemic regions. This is particularly important given that antibody longevity to the P. falciparum orthologs is unknown. No association was detected between recent $P$. falciparum infections and antibody levels to the SEMs, however the prevalence of $P$. falciparum infections in the validation cohorts was very low $(0.3-0.6 \%)$. Given the relatively low sequence homology between $P$. falciparum and $P$. vivax, extensive cross-reactivity is unlikely. This is however not necessarily the case for $P$. ovale, $P$. malariae or $P$. knowlesi which are more closely related to $P$. vivax (protein sequence identity for 
top eight antigens $13.7-83.4 \%$ ). On a programmatic level, limited cross-reactivity between $P$. vivax, $P$. falciparum, $P$. ovale or $P$. malariae may not be an issue in areas outside Africa. It has been well documented that rates of $P$. vivax relapse after $P$. falciparum episodes or infections are very high $(47,48)$, sometimes as high as following a $P$. vivax infection. This indicates that the presence of a $P$. falciparum infection is a marker of $P$. vivax hypnozoite carriage and it has therefore been suggested that in co-endemic areas all $P$. falciparum patients should receive anti$P$. vivax relapse therapy (47). The same is likely to apply to $P$. malariae and $P$. ovale infections, which regularly occur in mixed species infections (49), and of which the latter also has dormant hypnozoites. Given the marked difference in patterns of exposure to the zoonotic $P$. knowlesi (50), cross-reactivity between $P$. vivax and $P$. knowlesi would be significantly more problematic (51).

In summary, the novel SEMs for $P$. vivax can accurately predict recent infection within the last 9 months. As most hypnozoites in tropical regions are expected to relapse within 9 months of a previous blood-stage infections (52), these markers can indirectly identify the individuals with the highest risk of harbouring hypnozoites. Past efforts for developing serology as a measure of exposure to $P$. vivax have only detected historical changes over time (53); we have shown that a carefully selected panel of SEMs can specifically detect recent exposure and could be used in a programmatic setting ("surveillance as an intervention" (54)). Application of our SEMs for seroTAT holds promise for an effective elimination strategy using primaquine or tafenoquine to target dormant hypnozoites. Given the risk of haemolysis in G6PD-deficient individuals treated with primaquine or tafenoquine, our tool has the potential to ensure such elimination strategies are targeted and therefore a safer, more acceptable and more effective option in malaria-endemic communities.

\section{Materials and Methods}

\section{Study design}

The goal of the study was to identify then validate suitable candidate $P$. vivax proteins that could be used as serological markers of recent exposure to $P$. vivax infections. This study was conducted in two parts: i) an antigen discovery phase utilising samples from two longitudinal cohorts that followed symptomatic $P$. vivax malaria patients over 9 months, and ii) a validation phase utilising samples from three one-year long observational cohort studies. The sample size was therefore predefined by availability of suitable plasma samples with matching epidemiological and molecular data.

\section{Study populations: antigen discovery phase}

Patients with confirmed $P$. vivax malaria were enrolled from local malaria clinics and hospitals in two sites in 2014: Tha Song Yang, Tak Province, Thailand, and Manaus, Amazonas State, Brazil. After receiving anti-malarial drug treatment according to respective National Treatment Guidelines, and providing written informed consent and/or assent, blood samples were taken over a period of 9 months as previously described (24) (Fig. 1a, Table 1). Presence or absence of Plasmodium spp. parasites during follow-up was determined by both microscopy and quantitative PCR. Blood samples were collected at enrolment and week 1, then every 2 weeks for 6 months, then every month until the end of the study. A subset of enrolled participants who 
had no evidence of recurrent parasitaemia during follow-up was selected for antibody measurements ( $n=32$ Thai patients, $n=33$ Brazilian patients).

The Ethics Committee of the Faculty of Tropical Medicine, Mahidol University, Thailand, approved the Thai study (MUTM 2014-025-01 and 02). The Ethics Review Board of the Fundação de Medicina Tropical Dr. Heitor Vieira Dourado (FMT-HVD) (957.875/2014) approved the Brazilian antigen discovery study. The Human Research Ethics Committee (HREC) at the Walter and Eliza Hall Institute of Medical Research (WEHI) approved samples for use in Melbourne (\#14/02).

Study populations: validation phase

Yearlong observational cohort studies were conducted over 2013-2014 in three sites: Kanchanaburi/Ratchaburi provinces, Thailand (14), Manaus, Brazil (Monteiro et al., in preparation), and the island of Ngella, Solomon Islands (Quah et al., in preparation) (Fig. 1b, Table 1). A total of 999 volunteers were enrolled from Thailand and sampled every month over the yearlong cohort, with 14 active case detection visits performed in total. 829 volunteers attended the final visit. A total of 1,274 residents of all age groups were enrolled from Brazil and sampled every month over the year-long period, with 13 active case detection visits performed in total. 928 volunteers attended the final visit with plasma from 925 available. 1,111 children were enrolled from the Solomon Islands, with 860 used for final analysis of the cohort (after exclusion of children who withdrew, had inconsistent attendance or failed to meet other inclusion criteria). The children were sampled monthly, with 11 active case detection visits in total. Of the 860 children, 754 attended the final visit. For all three studies, at each visit volunteers completed a brief survey outlining their health over the past month (to determine the possibility of missed malarial infections), in addition to travel history and bed net usage. A finger-prick blood sample was also taken and axillary temperature recorded. Passive case detection was performed throughout the yearlong period.

The Ethics Committee of the Faculty of Tropical Medicine, Mahidol University, Thailand approved the Thai cohort (MUTM 2013-027-01). The Brazilian study was approved by the FMT-HVD (51536/2012), by the Brazilian National Committee of Ethics (CONEP) (349.211/2013) and by the Ethics Committee of the Hospital Clínic, Barcelona, Spain (2012/7306). The National Health Research and Ethics Committee of the Solomon Islands Ministry of Health and Medical Services (HRC12/022) approved the Solomon Islands study. The HREC at WEHI approved samples for use in Melbourne (\#14/02).

Study populations: malaria nä̈ve control panels

Three panels of malaria naïve control plasma samples were collected from individuals with no known recent exposure to malaria (Table 1). Samples were as follows: 102 individuals from the Volunteer Blood Donor Registry (VBDR) in Melbourne, Australia, 100 individuals from the Australian Red Cross (ARC), Melbourne, Australia, and 72 individuals from the Thai Red Cross (TRC), Bangkok, Thailand. Standard TRC screening procedures exclude individuals from donating blood if they have had a confirmed malaria infection within the previous 3 years or have travelled to malaria-endemic regions within the past year. The HREC at WEHI approved collection and/or use of these control samples (\#14/02).

\section{Procedures}


Blood samples were collected by finger prick into EDTA tubes. Blood was separated into packed red cells and plasma at the field site. Packed red cells were stored at $-20^{\circ} \mathrm{C}$ and plasma at $-80^{\circ} \mathrm{C}$ prior to use in molecular and serological assays, respectively. Malaria parasites were detected by qPCR as previously described $(55,56)$.

\section{Antibody measurements}

$P$. vivax malaria proteins were expressed using the wheat germ cell-free (WGCF) system (CellFree Sciences, Yokohama, Japan) at either Ehime University or CellFree Sciences. For the antigen discovery phase, $307 \mathrm{P}$. vivax protein constructs were expressed at a small-scale with a biotin tag and antibodies measured using the AlphaScreen ${ }^{\mathrm{TM}}$ assay, as previously described (24). Plasma samples from weeks zero (enrolment), 12, 24 and 36 in the subset of volunteers described above were used ( $n=32$ Thai patients, $n=33$ Brazilian patients). The raw AlphaScreen ${ }^{\mathrm{TM}}$ data was converted to relative antibody units based on plate-specific standard curves, with final units ranging from 0-400 (seropositivity was defined as a relative antibody unit greater than 0).

For the validation phase, down-selected proteins were produced at a high yield using dialysisbased refeeding WGCF methods and purified on an affinity matrix using a His-tag. The purified proteins were stored and shipped in the following buffer: $20 \mathrm{mM}$ Na-phosphate $\mathrm{pH} 7 \cdot 5,0 \cdot 3 \mathrm{M}$ $\mathrm{NaCl}, 500 \mathrm{mM}$ imidazole and $10 \%(\mathrm{v} / \mathrm{v})$ glycerol. Protein yields and purity were determined using SDS-PAGE followed by Coomassie Brilliant Blue staining using standard laboratory methods. An additional 20 P. vivax proteins known to be highly immunogenic were also included at the validation phase and were produced as previously described $(26-28,30,57,58)$ or using previously described methods (59) (see Table 2 and Table S2). The $P$. vivax proteins were then coupled to $\mathrm{COOH}$ microspheres as previously described (13). Protein-specific IgG antibody levels were measured in a multiplexed Luminex ${ }^{\circledR}$ assay as previously described (29). Plasma samples from the last visit of the three year-long observational cohort studies were used. Median fluorescent intensity (MFI) values from the Luminex ${ }^{\circledR}-200$ were converted to arbitrary relative antibody units based on a standard curve generated with a positive control plasma pool from highly immune adults from Papua New Guinea (PNG) (29).

\section{Statistical modelling - antigen discovery phase}

The change in measured antibody responses following $P$. vivax infection in patients in the antigen discovery phase was analysed using mixed-effects linear regression models, as previously described (24). Estimated half-lives for 307 P. vivax proteins were previously reported (24), with an additional 35 proteins included for this study. All estimated half-lives are provided in Supplementary Table S1. Antibody responses were measured at 0, 3, 6 and 9 months using the AlphaScreen ${ }^{\mathrm{TM}}$ assay, and the model was only fitted to individuals who were seropositive at baseline. Denote $A_{i j k}$ to be the antibody titre in participant $i$ to protein $j$ at time $t_{k}$ which can be described by the following linear model:

$$
\log \left(A_{i j k}\right) \sim\left(\log \left(\alpha_{j}^{0}\right)+\log \left(\alpha_{i j}\right)\right)+\left(r_{j}^{0}+r_{i j}\right) t_{k}+\varepsilon_{j}
$$

where $\alpha_{j}^{0}$ is the geometric mean titre (GMT) at the time of infection; $\log \left(\alpha_{i j}\right)$ is a random effect accounting for the difference between participant $i$ 's initial antibody titre and the population-level 
GMT; $r_{j}^{0}$ is the average rate of decay of antibodies to protein $j$ in the population; $r_{i j}$ is a random effect for the difference between the decay rate of individual $i$ with the population-level average; and $\varepsilon_{j} \sim \mathrm{N}\left(0, \sigma_{m, j}\right)$ is a Normally distributed error term. In particular it is assumed that the random effects for the initial antibody titres are Normally distributed: $\log \left(\alpha_{i j}\right) \sim \mathrm{N}\left(0, \sigma_{A, j}\right)$, and that the random effects for the variation in decay rates are also Normally distributed: $r_{i j} \sim \mathrm{N}(0$, $\left.\sigma_{r, j}\right)$.

The linear regression model for the decay of antibody titres described in equation (S1) has three sources of variation: (i) variation in initial antibody response following infection; (ii) between individual variation in antibody decay rate; and (iii) measurement error. Notably, all these sources of variations are described by Normal distributions so their combined variation will also be described by a Normal distribution. Therefore, $x_{i j}=\log \left(A_{i j}\right)$, the expected log antibody titre in individual $i$ to protein $j$ at time $t$, can be described by the following distribution:

$$
\mathbf{P}\left(x_{i j} \mid t\right)=\frac{1}{\sqrt{2 \pi\left(\sigma_{\alpha, j}^{2}+t^{2} \sigma_{r, j}^{2}+\sigma_{m, j}^{2}\right)}} e^{-\frac{\left(x_{i j}-\alpha_{j}^{0}-r_{j}^{0} t\right)^{2}}{2\left(\sigma_{\alpha, j}^{2}+t^{2} \sigma_{r, j}^{2}+\sigma_{m, j}^{2}\right)}}
$$

The probability distribution for the time since infection $t$ given measured antibody titre $x_{i j}$ can be calculated by inverting equation (1) using Bayes rule (60), allowing estimation of the probability that the time since last infection was less than 9 months.

It was possible to select $>10^{42}$ subsets from the panel of 142 prioritised SEMs in the antigen discovery phase. A simulated annealing algorithm was therefore used to explore this highdimensional space and select optimal combinations of proteins $(26,61)$. Combinations of proteins were chosen to optimise the likelihood that sampled antibody responses were correctly classified as having occurred within 9 months of infection.

\section{Statistical modelling - validation phase}

Individuals in the validation phase were classified into two categories depending on whether they had PCR-detectable blood-stage $P$. vivax infections within the 9 months prior to measurement of antibody responses (Table 1). In theory it is possible to provide quantitative estimates of the time since last infection, however a more useful outcome in practice is whether an individual has been infected within some past-time period. 9 months was selected due to the biology of $P$. vivax hypnozoite relapses as described in the Introduction (highest incidence of relapse within the first 9 months following mosquito bite infection (20)), and because this threshold fell within a time period for which data were available (the three longitudinal cohorts had follow-up for 12 months).

Initial classification performance of antibody responses to the 60 down-selected SEMs to classify recent $P$. vivax infections was assessed using a linear discriminant analysis (LDA). There were 1,770 ways of choosing two out of 60 proteins, 34,220 ways of choosing three proteins, 487,635 ways of choosing four proteins, and 5,461,512 ways of choosing five proteins. All combinations up to size four were exhaustively evaluated to optimise classification performance for the three 
sensitivity and specificity targets described in the Results. To investigate combinations of size five, the 100 best combinations of four proteins for each of the three TPPs were identified. All possible remaining proteins were added to each of these 300 combinations, and the classification performance of all of these combinations of size five was assessed. A similar procedure was implemented to investigate classification performance of combinations of proteins up to size eight. Fig. S6 shows an overview of classification performance.

After determining the highest ranking SEMs using the LDA classifier, the top eight candidates were further assessed using a range of other classification algorithms, including logistic regression, quadratic discriminant analysis (QDA), decision trees, and random forests (62). Decision trees were implemented using the rpart $\mathrm{R}$ package. Random forests were implemented using the randomForest $\mathrm{R}$ package. Two novel classification algorithms incorporating a statistical model of antibody decay over time were also developed (see Supplementary Materials and Methods). These algorithms were combined into a composite algorithm so that the optimal algorithm and selection of proteins was selected for each target of sensitivity and specificity. All classification algorithms were cross-validated using 500 randomly sampled, disjoint training and testing subsets.

\section{Data Availability}

All data is available within the figures and tables of the results and supplementary materials. Antibody half-lives and parameters used to generate Figure 3 are in Table S1.

\section{Code Availability}

Code for statistical modeling can be provided upon request.

\section{Supplementary Materials}

Materials and Methods

Fig. S1. Association between measured antibody titre and time since infection.

Fig. S2. Dynamics of multiple antibodies.

Fig. S3. Estimates of (A) antibody half-life, and (B) the variance of each antibody response for $104 P$. vivax proteins in the antigen discovery phase.

Fig. S4. Increasing the maximum number of proteins allowed in a panel leads to diminishing increases in likelihood and classification performance.

Fig. S5. Measured antibody responses to 60 proteins on the Luminex ${ }^{\circledR}$ platform, stratified by geographical location and time since last PCR detected infection.

Fig. S6. Performance of LDA classifier for combinations of proteins up to size eight for identifying individuals with blood-stage $P$. vivax infection within the last 9 months.

Fig S7. Network visualization of antigens.

Fig S8. Variable importance plot generated by a random forests algorithm for identify antigens that contribute to accurate classification.

Fig. S9. Receiver operating characteristic (ROC) curves depicting comparison of cross-validated classification performance for the seven classification algorithms considered. 
Fig. S10. Receiver operating characteristic (ROC) curve for the composite classification algorithm.

Fig. S11. Association between background reactivity in non-malaria exposed controls and ranking of candidate SEMs.

Fig. S12. Assessment of incorporating information on an individual's age into a Random Forests classifier.

Fig. S13. Assessment of the role of removing data from negative control participants on classification performance using a Random Forests classifier.

Fig. S14. Association between measurements of our top eight $P$. vivax proteins and time since last PCR detected blood-stage $P$. falciparum infection.

Table S1. Estimated antibody half-lives from antigen discovery phase.

Table S2. Purified $P$. vivax proteins used in the validation phase and their individual performance, complete list.

Table S3. Association of antibody level with current $P$. vivax infection.

\section{References and Notes:}

1. "World Malaria Report," (World Health Organisation, Geneva, Switzerland, 2017).

2. A. Waltmann, A. W. Darcy, I. Harris, C. Koepfli, J. Lodo, V. Vahi, D. Piziki, G. D. Shanks, A. E. Barry, M. Whittaker, J. W. Kazura, I. Mueller, High Rates of Asymptomatic, Sub-microscopic Plasmodium vivax Infection and Disappearing Plasmodium falciparum Malaria in an Area of Low Transmission in Solomon Islands. PLoS Negl Trop Dis 9, e0003758 (2015).

3. I. Mueller, M. R. Galinski, J. K. Baird, J. M. Carlton, D. K. Kochar, P. L. Alonso, H. A. del Portillo, Key gaps in the knowledge of Plasmodium vivax, a neglected human malaria parasite. Lancet Infect Dis 9, 555566 (2009).

4. C. M. Moreira, M. Abo-Shehada, R. N. Price, C. J. Drakeley, A systematic review of sub-microscopic Plasmodium vivax infection. Malar J 14, 360 (2015).

5. L. J. Robinson, R. Wampfler, I. Betuela, S. Karl, M. T. White, C. S. Li Wai Suen, N. E. Hofmann, B. Kinboro, A. Waltmann, J. Brewster, L. Lorry, N. Tarongka, L. Samol, M. Silkey, Q. Bassat, P. M. Siba, L. Schofield, I. Felger, I. Mueller, Strategies for understanding and reducing the Plasmodium vivax and Plasmodium ovale hypnozoite reservoir in Papua New Guinean children: a randomised placebo-controlled trial and mathematical model. PLoS Med 12, e1001891 (2015).

6. D. M. Parker, S. A. Matthews, G. Yan, G. Zhou, M. C. Lee, J. Sirichaisinthop, K. Kiattibutr, Q. Fan, P. Li, J. Sattabongkot, L. Cui, Microgeography and molecular epidemiology of malaria at the Thailand-Myanmar border in the malaria pre-elimination phase. Malar J 14, 198 (2015).

7. P. Mogeni, T. N. Williams, I. Omedo, D. Kimani, J. M. Ngoi, J. Mwacharo, R. Morter, C. Nyundo, J. Wambua, G. Nyangweso, M. Kapulu, G. Fegan, P. Bejon, Detecting Malaria Hotspots: A Comparison of Rapid Diagnostic Test, Microscopy, and Polymerase Chain Reaction. J Infect Dis 216, 1091-1098 (2017).

8. X. C. Ding, M. P. Ade, J. K. Baird, Q. Cheng, J. Cunningham, M. Dhorda, C. Drakeley, I. Felger, D. Gamboa, M. Harbers, S. Herrera, N. Lucchi, A. Mayor, I. Mueller, J. Sattabongkot, A. Ratsimbason, J. Richards, M. Tanner, I. J. Gonzalez, Defining the next generation of Plasmodium vivax diagnostic tests for control and elimination: Target product profiles. PLoS Negl Trop Dis 11, e0005516 (2017).

9. $\quad$ "Malaria surveillance, monitoring \& evaluation: a reference manual," (2018).

10. I. Harris, W. W. Sharrock, L. M. Bain, K. A. Gray, A. Bobogare, L. Boaz, K. Lilley, D. Krause, A. Vallely, M. L. Johnson, M. L. Gatton, G. D. Shanks, Q. Cheng, A large proportion of asymptomatic Plasmodium infections with low and sub-microscopic parasite densities in the low transmission setting of Temotu Province, Solomon Islands: challenges for malaria diagnostics in an elimination setting. Malar J 9, 254 (2010).

11. I. Sutanto, A. Kosasih, I. R. F. Elyazar, D. R. Simanjuntak, T. A. Larasati, M. S. Dahlan, I. Wahid, I. Mueller, C. Koepfli, R. Kusriastuti, A. Surya, F. J. Laihad, W. A. Hawley, F. H. Collins, J. K. Baird, N. F. 
Lobo, Negligible Impact of Mass Screening and Treatment on Meso-endemic Malaria Transmission at West Timor in Eastern Indonesia: A Cluster-Randomised Trial. Clin Infect Dis, (2018).

12. K. I. C. Ong, H. Kosugi, S. Thoeun, H. Araki, M. M. Thandar, M. Iwagami, B. Hongvanthong, P. T. Brey, S. Kano, M. Jimba, Systematic review of the clinical manifestations of glucose-6-phosphate dehydrogenase deficiency in the Greater Mekong Subregion: implications for malaria elimination and beyond. BMJ Glob Health 2, e000415 (2017).

13. R. J. Longley, C. T. Franca, M. T. White, C. Kumpitak, P. Sa-Angchai, J. Gruszczyk, J. B. Hostetler, A. Yadava, C. L. King, R. M. Fairhurst, J. C. Rayner, W. H. Tham, W. Nguitragool, J. Sattabongkot, I. Mueller, Asymptomatic Plasmodium vivax infections induce robust IgG responses to multiple blood-stage proteins in a low-transmission region of western Thailand. Malar J 16, 178 (2017).

14. R. J. Longley, A. Reyes-Sandoval, E. Montoya-Diaz, S. Dunachie, C. Kumpitak, W. Nguitragool, I. Mueller, J. Sattabongkot, Acquisition and Longevity of Antibodies to Plasmodium vivax Preerythrocytic Antigens in Western Thailand. Clin Vaccine Immunol 23, 117-124 (2016).

15. D. A. Helb, K. K. Tetteh, P. L. Felgner, J. Skinner, A. Hubbard, E. Arinaitwe, H. Mayanja-Kizza, I. Ssewanyana, M. R. Kamya, J. G. Beeson, J. Tappero, D. L. Smith, P. D. Crompton, P. J. Rosenthal, G. Dorsey, C. J. Drakeley, B. Greenhouse, Novel serologic biomarkers provide accurate estimates of recent Plasmodium falciparum exposure for individuals and communities. Proc Natl Acad Sci U S A 112, E44384447 (2015).

16. R. J. Welch, B. L. Anderson, C. M. Litwin, Rapid immunochromatographic strip test for detection of anti$\mathrm{K} 39$ immunoglobulin $\mathrm{G}$ antibodies for diagnosis of visceral leishmaniasis. Clin Vaccine Immunol 15, 14831484 (2008).

17. S. Gwyn, A. Mitchell, D. Dean, H. Mkocha, S. Handali, D. L. Martin, Lateral flow-based antibody testing for Chlamydia trachomatis. J Immunol Methods 435, 27-31 (2016).

18. M. L. Landry, Diagnostic tests for influenza infection. Curr Opin Pediatr 23, 91-97 (2011).

19. B. Greenhouse, D. L. Smith, I. Rodriguez-Barraquer, I. Mueller, C. Drakeley, Taking sharper pictures of malaria with CAMERAs: Combined Antibodies to Measure Exposure Recency Assays. bioRxiv, (2018).

20. N. J. White, Determinants of relapse periodicity in Plasmodium vivax malaria. Malar J 10, 297 (2011).

21. K. E. Battle, M. S. Karhunen, S. Bhatt, P. W. Gething, R. E. Howes, N. Golding, T. P. Van Boeckel, J. P. Messina, G. D. Shanks, D. L. Smith, J. K. Baird, S. I. Hay, Geographical variation in Plasmodium vivax relapse. Malar J 13, 144 (2014).

22. M. T. White, G. Shirreff, S. Karl, A. C. Ghani, I. Mueller, Variation in relapse frequency and the transmission potential of Plasmodium vivax malaria. Proc Biol Sci 283, 20160048 (2016).

23. J. M. Carlton, J. H. Adams, J. C. Silva, S. L. Bidwell, H. Lorenzi, E. Caler, J. Crabtree, S. V. Angiuoli, E. F. Merino, P. Amedeo, Q. Cheng, R. M. Coulson, B. S. Crabb, H. A. Del Portillo, K. Essien, T. V. Feldblyum, C. Fernandez-Becerra, P. R. Gilson, A. H. Gueye, X. Guo, S. Kang'a, T. W. Kooij, M. Korsinczky, E. V. Meyer, V. Nene, I. Paulsen, O. White, S. A. Ralph, Q. Ren, T. J. Sargeant, S. L. Salzberg, C. J. Stoeckert, S. A. Sullivan, M. M. Yamamoto, S. L. Hoffman, J. R. Wortman, M. J. Gardner, M. R. Galinski, J. W. Barnwell, C. M. Fraser-Liggett, Comparative genomics of the neglected human malaria parasite Plasmodium vivax. Nature 455, 757-763 (2008).

24. R. J. Longley, M. T. White, E. Takashima, M. Morita, B. N. Kanoi, C. S. N. Li Wai Suen, I. Betuela, A. Kuehn, P. Sripoorote, C. T. Franca, P. Siba, L. J. Robinson, M. Lacerda, J. Sattabongkot, T. Tsuboi, I. Mueller, Naturally acquired antibody responses to more than 300 Plasmodium vivax proteins in three geographic regions. PLoS Negl Trop Dis 11, e0005888 (2017).

25. T. Tsuboi, S. Takeo, H. Iriko, L. Jin, M. Tsuchimochi, S. Matsuda, E. T. Han, H. Otsuki, O. Kaneko, J. Sattabongkot, R. Udomsangpetch, T. Sawasaki, M. Torii, Y. Endo, Wheat germ cell-free system-based production of malaria proteins for discovery of novel vaccine candidates. Infect Immun 76, 1702-1708 (2008).

26. C. T. Franca, M. T. White, W. Q. He, J. B. Hostetler, J. Brewster, G. Frato, I. Malhotra, J. Gruszczyk, C. Huon, E. Lin, B. Kiniboro, A. Yadava, P. Siba, M. R. Galinski, J. Healer, C. Chitnis, A. F. Cowman, E. Takashima, T. Tsuboi, W. H. Tham, R. M. Fairhurst, J. C. Rayner, C. L. King, I. Mueller, Identification of highly-protective combinations of Plasmodium vivax recombinant proteins for vaccine development. Elife 6, (2017).

27. C. T. Franca, W. Q. He, J. Gruszczyk, N. T. Lim, E. Lin, B. Kiniboro, P. M. Siba, W. H. Tham, I. Mueller, Plasmodium vivax Reticulocyte Binding Proteins Are Key Targets of Naturally Acquired Immunity in Young Papua New Guinean Children. PLoS Negl Trop Dis 10, e0005014 (2016). 
28. F. Lu, J. Li, B. Wang, Y. Cheng, D. H. Kong, L. Cui, K. S. Ha, J. Sattabongkot, T. Tsuboi, E. T. Han, Profiling the humoral immune responses to Plasmodium vivax infection and identification of candidate immunogenic rhoptry-associated membrane antigen (RAMA). J Proteomics 102, 66-82 (2014).

29. C. T. Franca, J. B. Hostetler, S. Sharma, M. T. White, E. Lin, B. Kiniboro, A. Waltmann, A. W. Darcy, C. S. Li Wai Suen, P. Siba, C. L. King, J. C. Rayner, R. M. Fairhurst, I. Mueller, An Antibody Screen of a Plasmodium vivax Antigen Library Identifies Novel Merozoite Proteins Associated with Clinical Protection. PLoS Negl Trop Dis 10, e0004639 (2016).

30. J. Gruszczyk, U. Kanjee, L. J. Chan, S. Menant, B. Malleret, N. T. Y. Lim, C. Q. Schmidt, Y. F. Mok, K. M. Lin, R. D. Pearson, G. Rangel, B. J. Smith, M. J. Call, M. P. Weekes, M. D. W. Griffin, J. M. Murphy, J. Abraham, K. Sriprawat, M. J. Menezes, M. U. Ferreira, B. Russell, L. Renia, M. T. Duraisingh, W. H. Tham, Transferrin receptor 1 is a reticulocyte-specific receptor for Plasmodium vivax. Science 359, 48-55 (2018).

31. I. S. Soares, J. W. Barnwell, M. U. Ferreira, M. Gomes Da Cunha, J. P. Laurino, B. A. Castilho, M. M. Rodrigues, A Plasmodium vivax vaccine candidate displays limited allele polymorphism, which does not restrict recognition by antibodies. Mol Med 5, 459-470 (1999).

32. B. Wang, F. Lu, Y. Cheng, J. H. Chen, H. Y. Jeon, K. S. Ha, J. Cao, M. H. Nyunt, J. H. Han, S. K. Lee, M. P. Kyaw, J. Sattabongkot, E. Takashima, T. Tsuboi, E. T. Han, Immunoprofiling of the tryptophan-rich antigen family in Plasmodium vivax. Infect Immun 83, 3083-3095 (2015).

33. Y. Cheng, B. Wang, S. Changrob, J. H. Han, J. Sattabongkot, K. S. Ha, P. Chootong, F. Lu, J. Cao, M. H. Nyunt, W. S. Park, S. H. Hong, C. S. Lim, T. Tsuboi, E. T. Han, Naturally acquired humoral and cellular immune responses to Plasmodium vivax merozoite surface protein 8 in patients with $\mathrm{P}$. vivax infection. Malar J 16, 211 (2017).

34. J. Hester, E. R. Chan, D. Menard, O. Mercereau-Puijalon, J. Barnwell, P. A. Zimmerman, D. Serre, De novo assembly of a field isolate genome reveals novel Plasmodium vivax erythrocyte invasion genes. PLoS Negl Trop Dis 7, e2569 (2013).

35. J. Jiang, J. W. Barnwell, E. V. Meyer, M. R. Galinski, Plasmodium vivax merozoite surface protein-3 (PvMSP3): expression of an 11 member multigene family in blood-stage parasites. PLoS One 8, e63888 (2013).

36. E. H. Ntege, E. Takashima, M. Morita, H. Nagaoka, T. Ishino, T. Tsuboi, Blood-stage malaria vaccines: post-genome strategies for the identification of novel vaccine candidates. Expert Rev Vaccines 16, 769-779 (2017).

37. R. N. Rabinovich, C. Drakeley, A. A. Djimde, B. F. Hall, S. I. Hay, J. Hemingway, D. C. Kaslow, A. Noor, F. Okumu, R. Steketee, M. Tanner, T. N. C. Wells, M. A. Whittaker, E. A. Winzeler, D. F. Wirth, K. Whitfield, P. L. Alonso, malERA: An updated research agenda for malaria elimination and eradication. PLoS Med 14, e1002456 (2017).

38. J. Cook, H. Reid, J. Iavro, M. Kuwahata, G. Taleo, A. Clements, J. McCarthy, A. Vallely, C. Drakeley, Using serological measures to monitor changes in malaria transmission in Vanuatu. Malar J 9, 169 (2010).

39. S. Ladeia-Andrade, M. U. Ferreira, K. K. Scopel, E. M. Braga, S. Bastos Mda, G. Wunderlich, J. R. Coura, Naturally acquired antibodies to merozoite surface protein (MSP)-1(19) and cumulative exposure to Plasmodium falciparum and Plasmodium vivax in remote populations of the Amazon Basin of Brazil. Mem Inst Oswaldo Cruz 102, 943-951 (2007).

40. J. Cook, N. Speybroeck, T. Sochanta, H. Somony, M. Sokny, F. Claes, K. Lemmens, M. Theisen, I. S. Soares, U. D'Alessandro, M. Coosemans, A. Erhart, Sero-epidemiological evaluation of changes in Plasmodium falciparum and Plasmodium vivax transmission patterns over the rainy season in Cambodia. Malar J 11, 86 (2012).

41. M. T. White, S. Karl, C. Koepfli, R. J. Longley, N. E. Hofmann, R. Wampfler, I. Felger, T. Smith, W. Nguitragool, J. Sattabongkot, L. Robinson, A. Ghani, I. Mueller, Plasmodium vivax and Plasmodium falciparum infection dynamics: re-infections, recrudescences and relapses. Malar J 17, 170 (2018).

42. M. T. White, J. T. Griffin, O. Akpogheneta, D. J. Conway, K. A. Koram, E. M. Riley, A. C. Ghani, Dynamics of the antibody response to Plasmodium falciparum infection in African children. J Infect Dis 210, 1115-1122 (2014).

43. M. J. Boyle, L. Reiling, F. H. Osier, F. J. Fowkes, Recent insights into humoral immunity targeting Plasmodium falciparum and Plasmodium vivax malaria. Int J Parasitol 47, 99-104 (2017).

44. J. S. Richards, D. I. Stanisic, F. J. Fowkes, L. Tavul, E. Dabod, J. K. Thompson, S. Kumar, C. E. Chitnis, D. L. Narum, P. Michon, P. M. Siba, A. F. Cowman, I. Mueller, J. G. Beeson, Association between 
naturally acquired antibodies to erythrocyte-binding antigens of Plasmodium falciparum and protection from malaria and high-density parasitemia. Clin Infect Dis 51, e50-60 (2010).

45. C. Arama, J. Skinner, D. Doumtabe, S. Portugal, T. M. Tran, A. Jain, B. Traore, O. K. Doumbo, D. H. Davies, M. Troye-Blomberg, A. Dolo, P. L. Felgner, P. D. Crompton, Genetic Resistance to Malaria Is Associated With Greater Enhancement of Immunoglobulin (Ig)M Than IgG Responses to a Broad Array of Plasmodium falciparum Antigens. Open Forum Infect Dis 2, ofv118 (2015).

46. T. Woodberry, G. Minigo, K. A. Piera, J. C. Hanley, H. D. de Silva, E. Salwati, E. Kenangalem, E. Tjitra, R. L. Coppel, R. N. Price, N. M. Anstey, M. Plebanski, Antibodies to Plasmodium falciparum and Plasmodium vivax merozoite surface protein 5 in Indonesia: species-specific and cross-reactive responses. J Infect Dis 198, 134-142 (2008).

47. N. M. Douglas, F. Nosten, E. A. Ashley, L. Phaiphun, M. van Vugt, P. Singhasivanon, N. J. White, R. N. Price, Plasmodium vivax recurrence following falciparum and mixed species malaria: risk factors and effect of antimalarial kinetics. Clin Infect Dis 52, 612-620 (2011).

48. S. Looareesuwan, N. J. White, S. Chittamas, D. Bunnag, T. Harinasuta, High rate of Plasmodium vivax relapse following treatment of falciparum malaria in Thailand. Lancet 2, 1052-1055 (1987).

49. I. Mueller, P. A. Zimmerman, J. C. Reeder, Plasmodium malariae and Plasmodium ovale--the "bashful" malaria parasites. Trends Parasitol 23, 278-283 (2007).

50. K. M. Fornace, L. S. Herman, T. R. Abidin, T. H. Chua, S. Daim, P. J. Lorenzo, L. Grignard, N. A. Nuin, L. T. Ying, M. J. Grigg, T. William, F. Espino, J. Cox, K. K. A. Tetteh, C. J. Drakeley, Exposure and infection to Plasmodium knowlesi in case study communities in Northern Sabah, Malaysia and Palawan, The Philippines. PLoS Negl Trop Dis 12, e0006432 (2018).

51. L. S. Herman, K. Fornace, J. Phelan, M. J. Grigg, N. M. Anstey, T. William, R. W. Moon, M. J. Blackman, C. J. Drakeley, K. K. A. Tetteh, Identification and validation of a novel panel of Plasmodium knowlesi biomarkers of serological exposure. PLoS Negl Trop Dis 12, e0006457 (2018).

52. $\quad$ N. J. White, M. Imwong, Relapse. Adv Parasitol 80, 113-150 (2012).

53. C. J. Drakeley, P. H. Corran, P. G. Coleman, J. E. Tongren, S. L. McDonald, I. Carneiro, R. Malima, J. Lusingu, A. Manjurano, W. M. Nkya, M. M. Lemnge, J. Cox, H. Reyburn, E. M. Riley, Estimating medium- and long-term trends in malaria transmission by using serological markers of malaria exposure. Proc Natl Acad Sci U S A 102, 5108-5113 (2005).

54. P. L. Alonso, G. Brown, M. Arevalo-Herrera, F. Binka, C. Chitnis, F. Collins, O. K. Doumbo, B. Greenwood, B. F. Hall, M. M. Levine, K. Mendis, R. D. Newman, C. V. Plowe, M. H. Rodriguez, R. Sinden, L. Slutsker, M. Tanner, A research agenda to underpin malaria eradication. PLoS Med 8, e1000406 (2011).

55. R. Wampfler, F. Mwingira, S. Javati, L. Robinson, I. Betuela, P. Siba, H. P. Beck, I. Mueller, I. Felger, Strategies for detection of Plasmodium species gametocytes. PLoS One 8, e76316 (2013).

56. A. Rosanas-Urgell, D. Mueller, I. Betuela, C. Barnadas, J. Iga, P. A. Zimmerman, H. A. del Portillo, P. Siba, I. Mueller, I. Felger, Comparison of diagnostic methods for the detection and quantification of the four sympatric Plasmodium species in field samples from Papua New Guinea. Malar J 9, 361 (2010).

57. J. Hietanen, A. Chim-Ong, T. Chiramanewong, J. Gruszczyk, W. Roobsoong, W. H. Tham, J. Sattabongkot, W. Nguitragool, Gene Models, Expression Repertoire, and Immune Response of Plasmodium vivax Reticulocyte Binding Proteins. Infect Immun 84, 677-685 (2015).

58. J. L. Cole-Tobian, P. Michon, M. Biasor, J. S. Richards, J. G. Beeson, I. Mueller, C. L. King, Strainspecific duffy binding protein antibodies correlate with protection against infection with homologous compared to heterologous plasmodium vivax strains in Papua New Guinean children. Infect Immun 77, 4009-4017 (2009).

59. J. Healer, J. K. Thompson, D. T. Riglar, D. W. Wilson, Y. H. Chiu, K. Miura, L. Chen, A. N. Hodder, C. A. Long, D. S. Hansen, J. Baum, A. F. Cowman, Vaccination with conserved regions of erythrocyte-binding antigens induces neutralizing antibodies against multiple strains of Plasmodium falciparum. PLoS One $\mathbf{8}$, e72504 (2013).

$60 . \quad$ B. Borremans, N. Hens, P. Beutels, H. Leirs, J. Reijniers, Estimating Time of Infection Using Prior Serological and Individual Information Can Greatly Improve Incidence Estimation of Human and Wildlife Infections. PLoS Comput Biol 12, e1004882 (2016).

61. S. Kirkpatrick, C. D. Gelatt, Jr., M. P. Vecchi, Optimization by simulated annealing. Science 220, 671-680 (1983).

62. T. Hastie, R. Tibshirani, J. Friedman, The elements of statistical learning: data mining, inference, and prediction. (Springer, New York, ed. 2nd, 2009). 
Acknowledgments: We gratefully acknowledge the extensive field teams that contributed to sample collection and qPCR assays, including Andrea Kuehn, Yi Wan Quah, Piyarat Sripoorote, and Andrea Waltmann. We thank all the individuals that participated in each of the studies, and thank the Australian and Thai Red Cross for donation of plasma samples. We thank the Volunteer Blood Donor Registry at WEHI for donation of plasma samples, and Lina Laskos and Jenni Harris for their collection and advice. We thank Christopher King (Case Western Reserve University) for provision of the PNG control plasma pool. Melanie Bahlo (Walter and Eliza Hall Institute) is thanked for her advice on algorithm development. Funding: We acknowledge funding from the Global Health Innovative Technology Fund (T2015-142 to IM), the National Institute of Allergy and Infectious Diseases (NIH grant 5R01 AI 104822 to JS and 5U19AI089686-06 to JK) and the National Health and Medical Research Council Australia (\#1092789 and \#1134989 to IM and \#1143187 to W-HT). Additional funding directly supporting field studies was from the TransEPI consortium (supported by the Bill and Melinda Gates Foundation). This work has been supported by FIND with funding from the Australian and British governments. We also acknowledge support from the National Research Council of Thailand. This work was made possible through Victorian State Government Operational Infrastructure Support and Australian Government NHMRC IRIISS. IM is supported by an NHMRC Senior Research Fellowship (1043345). DLD is supported by an NHMRC Principal Research Fellowship (1023636). TT was supported in part by JSPS KAKENHI (JP15H05276, JP16K15266) in Japan. W.H.T. is a Howard Hughes Medical Institute-Wellcome Trust International Research Scholar (208693/Z/17/Z). Author contributions: RJL, MW, TT and IM designed the study. WN, WM, JK, ML, JS and IM conducted the cohort studies. ET, MM, MH, FM, W-HT, JH, CH, CEC and TT expressed the proteins. RJL, ET, MM, JB, and S-JL performed antibody measurements. RJL, CSNL-W-S and MW did the data management and analysis. MW performed modelling. RJL, MW and IM wrote the draft of the report. LJR, CP, DLD, XCD and IJG provided expert advice. All authors contributed to data interpretation and revision of the report. Competing interests: FIND contributed to early funding of this work and had a role in data interpretation, writing of the report and the decision to submit. No other funders of this study had any role in study design, data collection, data analysis, data interpretation, writing of the report, and the decision to submit. RJL, MW, TT and IM are inventors on patent PCT/US17/67926 on a system, method, apparatus and diagnostic test for Plasmodium vivax. 


\section{Figures:}

\section{(A) Antigen discovery data}

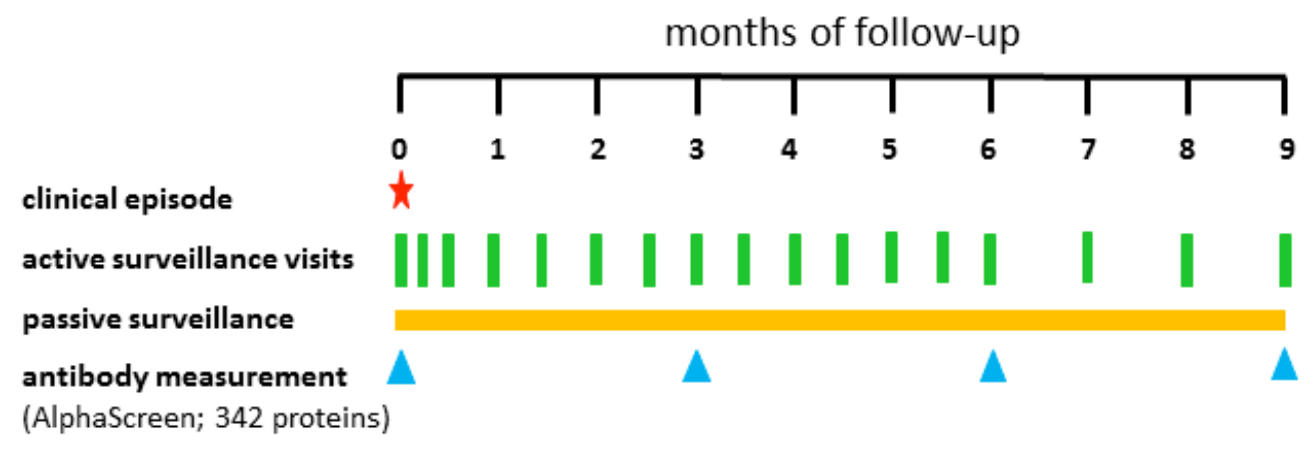

(B) Validation data

months of follow-up

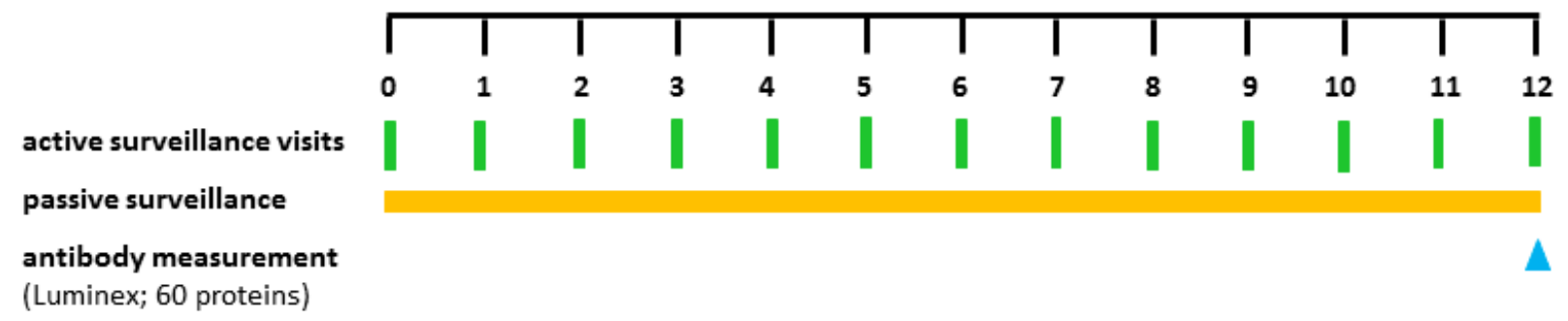

Fig. 1. Study design and follow-up schedule. (A) Thai and Brazilian patients were enrolled following a clinical episode of $P$. vivax and treated according to the relevant National Guidelines, with treatment directly observed (DOT) to ensure compliance and reduced risk of relapse. Volunteers were followed for 9 months after enrolment, with finger-prick blood samples collected at enrolment and week 1, then every 2 weeks for 6 months, then every month. Antibody levels were measured in a subset of 32 Thai and 33 Brazilian volunteers who were confirmed to be free of blood-stage Plasmodium parasites by analysing all samples by light microscopy and qPCR. (B) 999 participants from Thailand, 1274 participants from Brazil, and 860 participants from the Solomon Islands were followed longitudinally for 12 months with active surveillance visits every month. Antibody levels were measured in plasma samples from the last visit. 


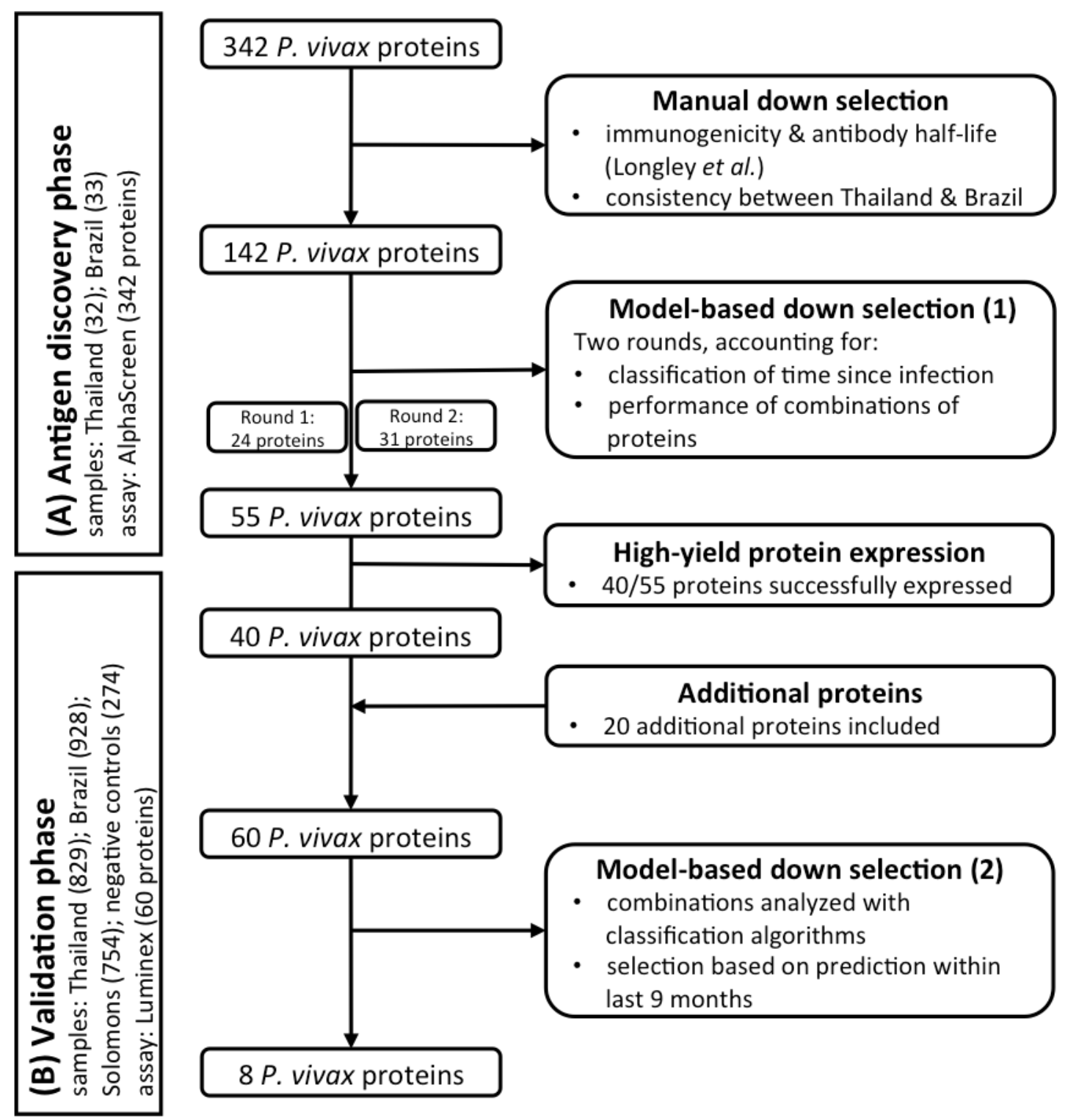

Fig. 2. Flow diagram of antigen down-selection pipeline. The studies and samples used are listed to the left in (A) and (B), whereas the pipeline and number of proteins included at each stage are listed to the right. 


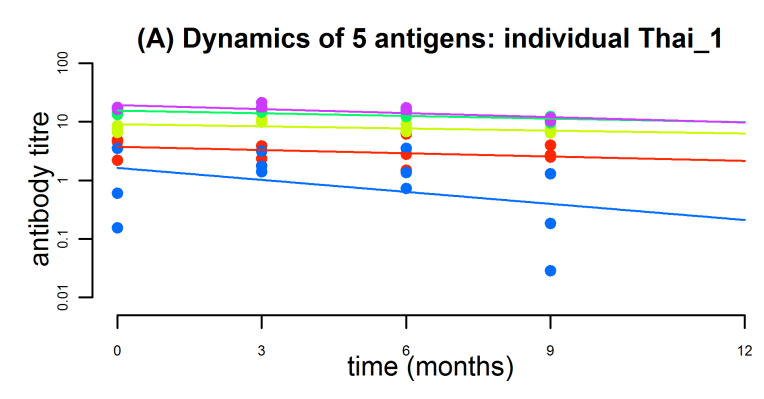

(C) Estimating infection time: individual Thai_1

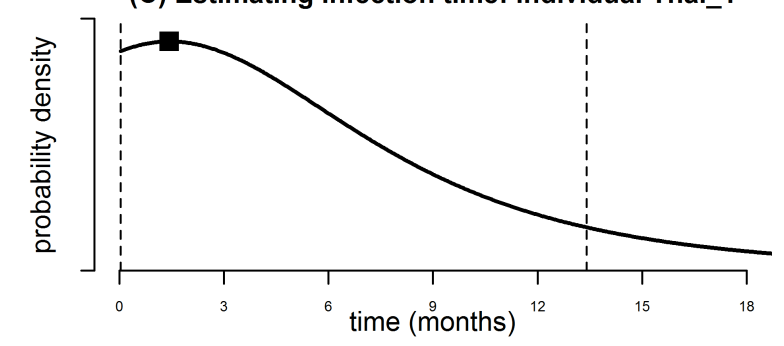

(E) Probability of antigen inclusion
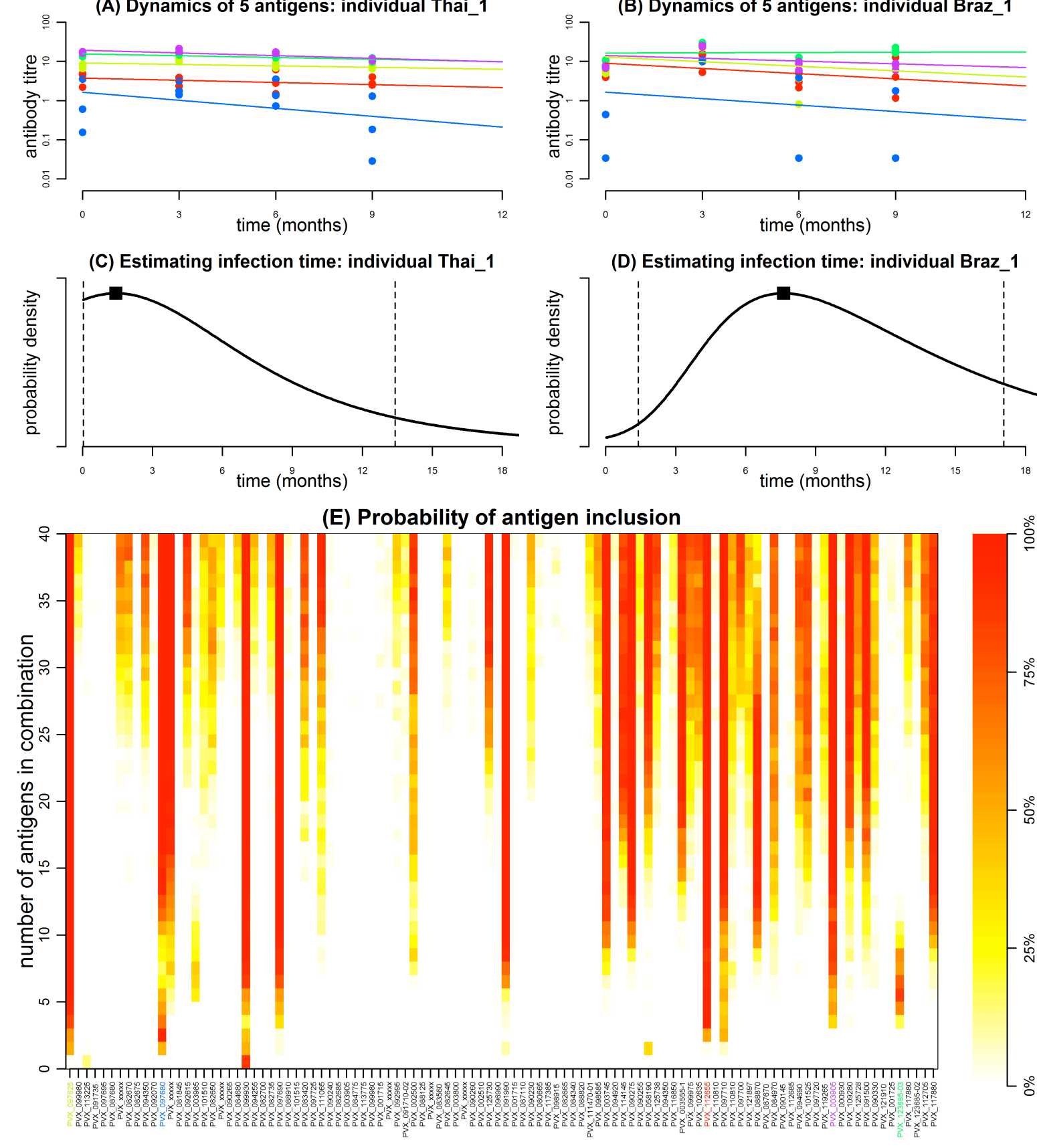

(D) Estimating infection time: individual Braz_1
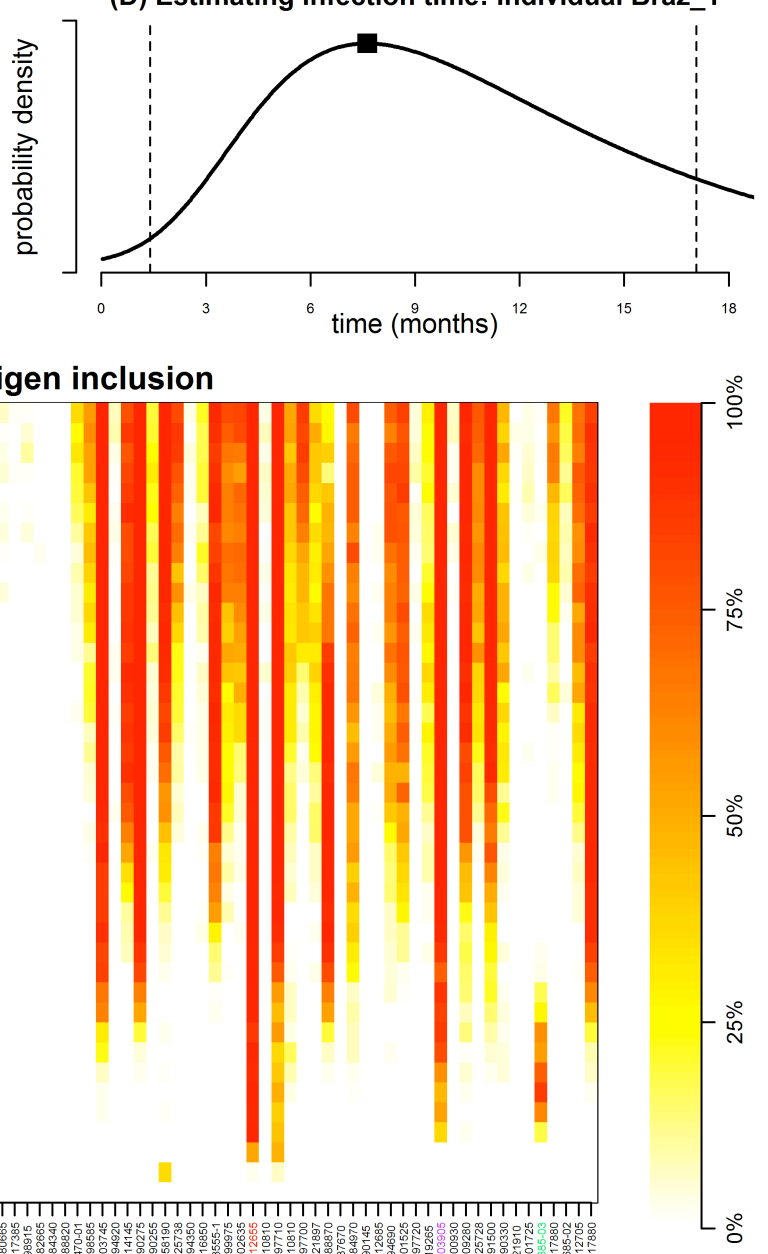

Fig. 3. Antigen discovery phase: estimating time since last $P$. vivax infection. (A, B) Examples of the dynamics of antibody responses to five proteins in individuals from Thailand and Brazil. Points represent measured antibody responses in triplicate on the AlphaScreen ${ }^{\mathrm{TM}}$ platform, and solid lines depict the fit of mixed-effects linear regression models. Each colour corresponds to one of the five proteins (PVX_097625, PVX_097680, PVX_112655, PVX_003905, PVX_123685-03) selected to provide optimal classification performance. $(\bar{C}, \mathrm{D})$ Using data on measured antibody responses 6 months after infection, the black curve shows the model 
estimated time since infection using the antibody responses shown in (A, B). The square point denotes the most likely estimate and dashed vertical lines denote the $95 \%$ confidence interval. In both cases the confidence intervals span 6 months. (E) Results of simulated annealing algorithm for estimating the probability that combinations of antigens predict time since last infection in the antigen discovery data sets. Colour is representative of the inclusion probability (red $100 \%$, white $0 \%$ ). The five proteins with data shown in figures a-d are highlighted in colour in panel e. These results came from the second round of down-selection on 104 P. vivax proteins (see Supplementary Methods for further details).

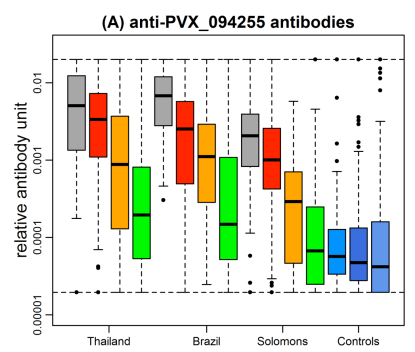

(E) anti-PVX_097625 antibodies
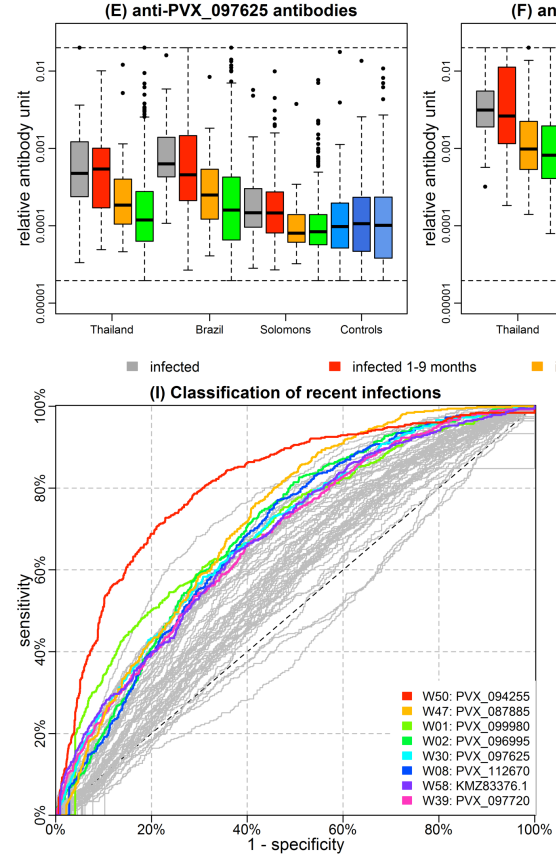

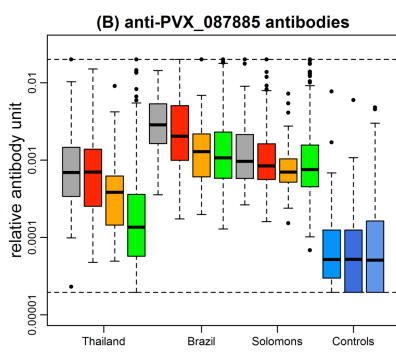

(F) anti-PVX_112670 antibodies

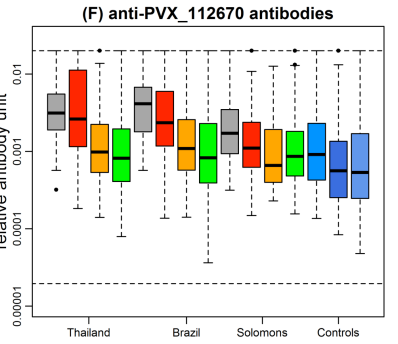

- infected 9-12 months $=$ no detected infection (J) Correlation between antibody titres

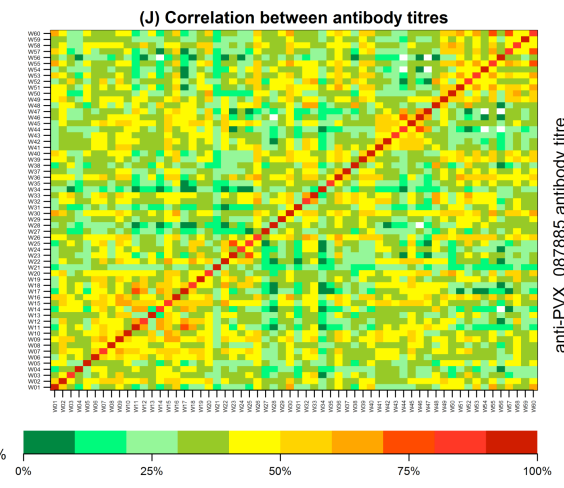

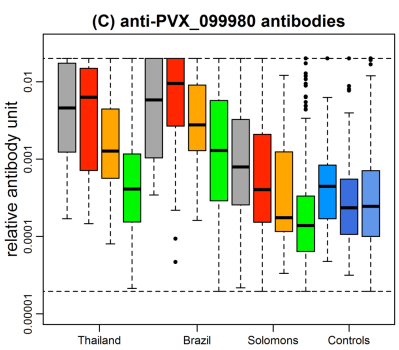

(G) anti-KMZ83376.1 antibodies
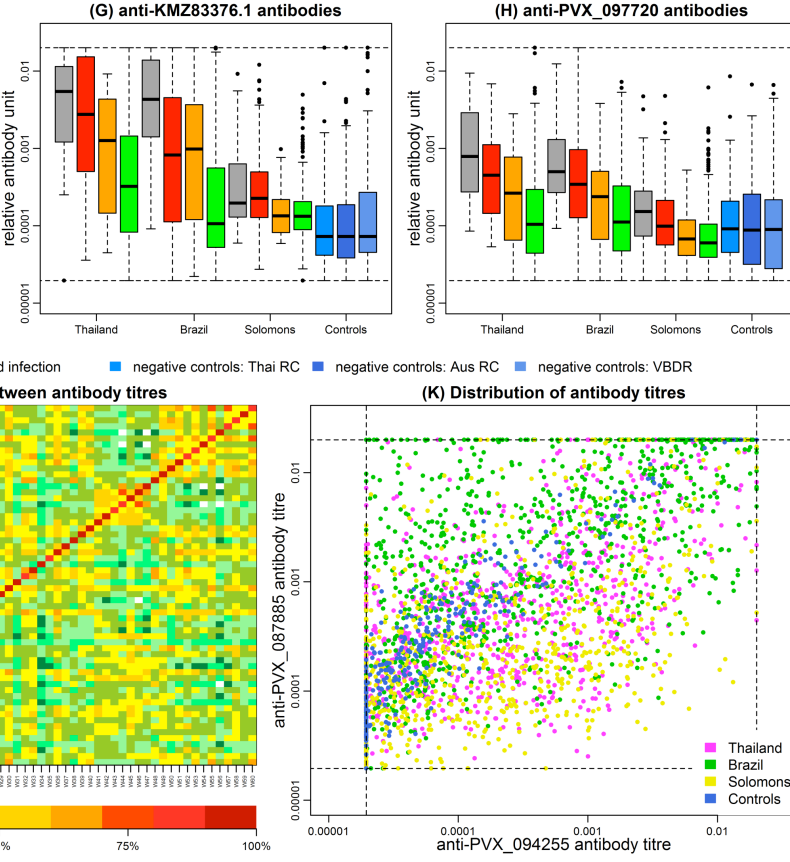

Fig. 4. Validation phase. (A-H) Measured antibody response to 8 antigens on the Luminex ${ }^{\circledR}$ platform, stratified by geographical location and time since last PCR detected infection. (I) Receiver operating characteristic (ROC) curve for classifying individuals as infected within the last 9 months using a threshold antibody titre to a single antigen. Coloured curves correspond to those proteins in panels a-h. (J) Spearman correlation between antibody titres. Correlation coefficients are indicating by the colour scale, with red being $100 \%$ correlation and dark green 0\% correlation. (K) 2-dimensional distribution of anti-PVX_099980 and anti-PVX_094255 antibody titres. 

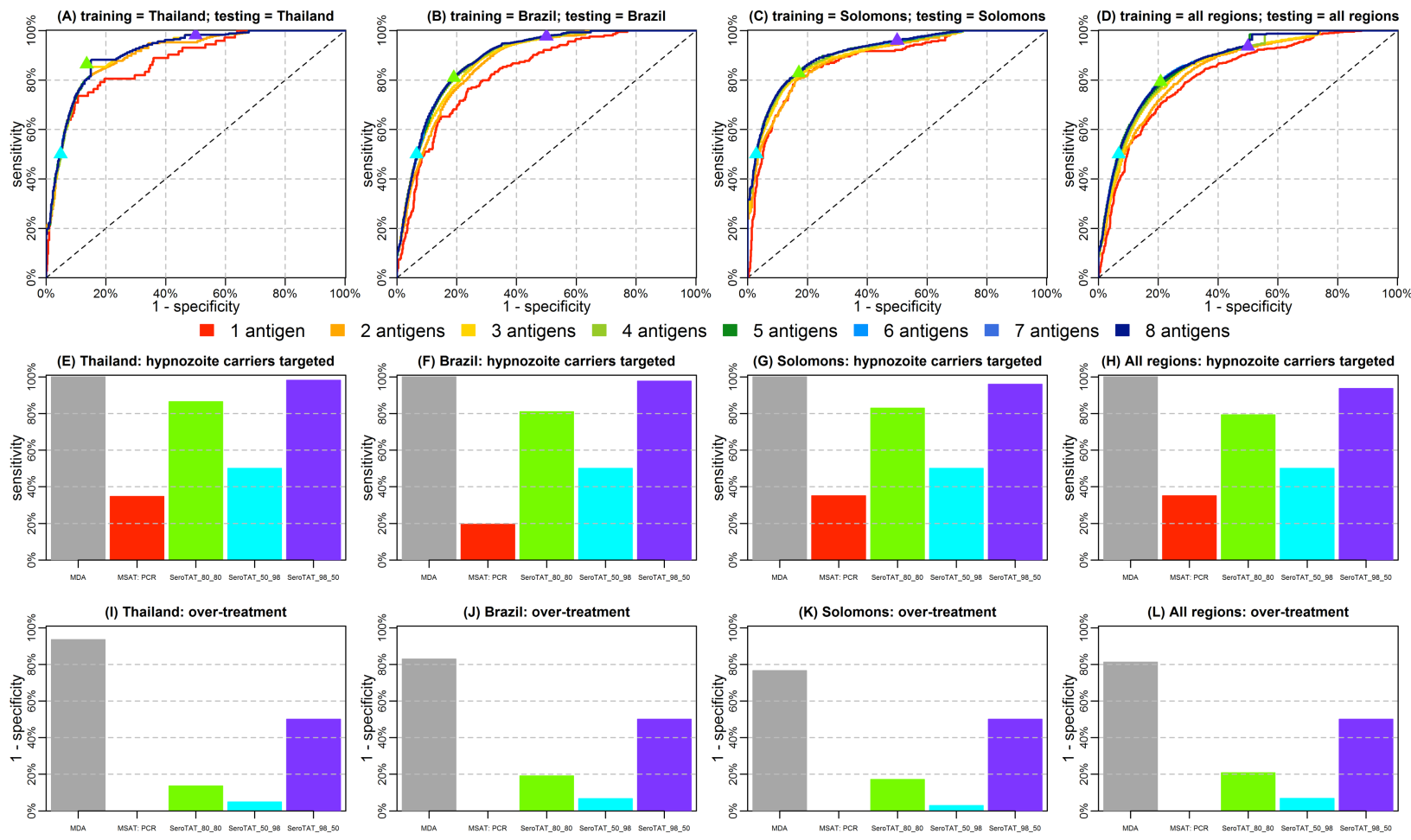

MDA

- MSAT: PCR

SeroTAT: $80 \%, 80 \%$ SeroTAT: $50 \%, 98 \%$

SeroTAT: $98 \%, 50 \%$

Fig. 5. Performance of the top $8 P$. vivax proteins for classifying recent infection and modelled ability to identify hypnozoites carriers. Cross-validated ROC curves generated from the composite algorithm classifier for identifying individuals with PCR detected infection in the last 9 months in (A) Thailand; (B) Brazil; (C) The Solomon Islands; and (D) all regions combined. The coloured triangles denote three different sensitivity and specificity targets for serological screening and treatment (SeroTAT) strategies. (E-H) Number of individuals targeted for primaquine treatment under a range of mass treatment strategies. (I-L) Proportion of individuals who had no exposure to $P$. vivax during the previous 9 months who would have been administered primaquine. 


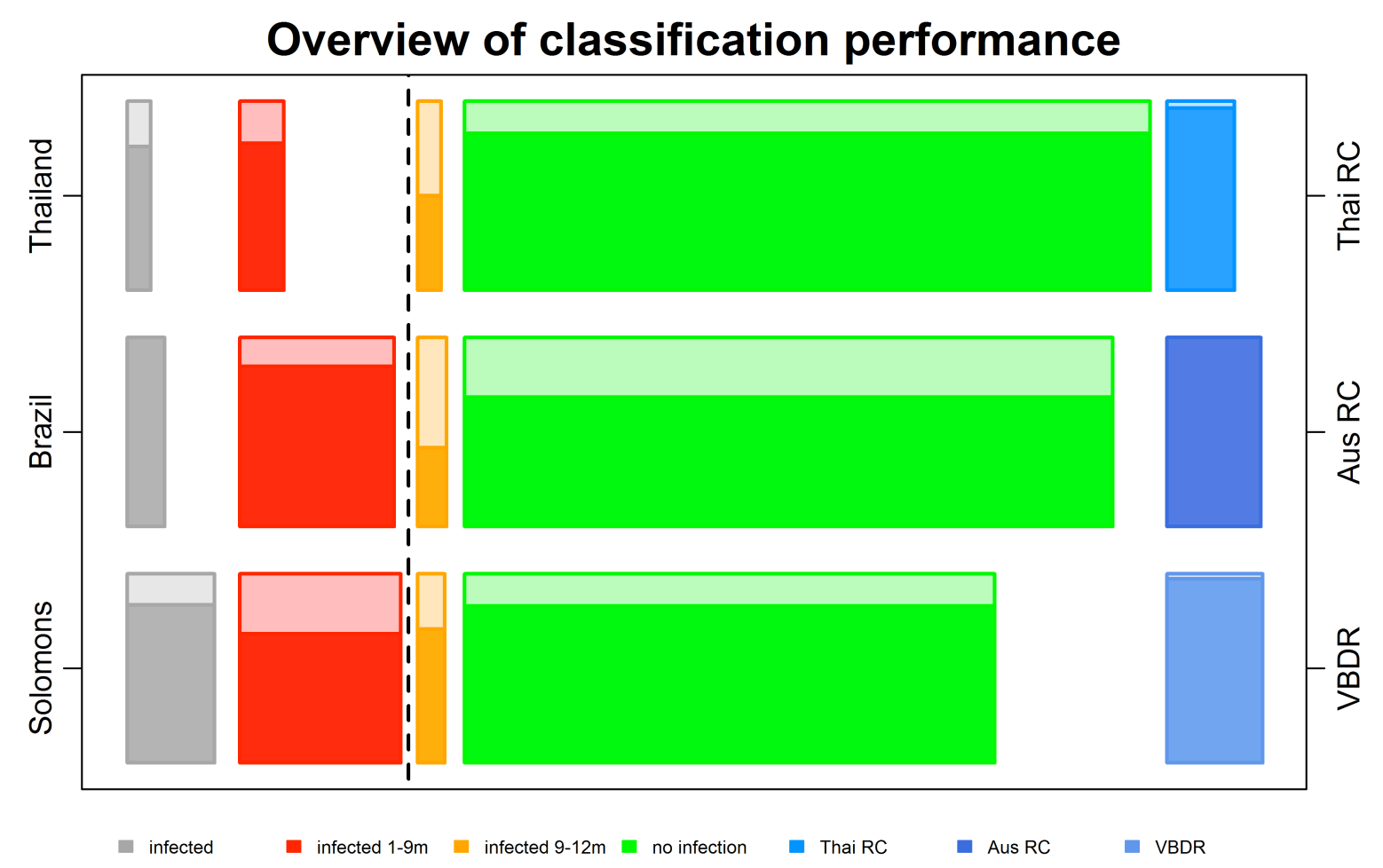

Fig. 6. Breakdown of classification performance of the composite algorithm with target sensitivity and specificity of $80 \%$. The size of each rectangle is proportional to the number of samples in each category (See Table 1). The coloured area represents the proportion correctly classified, and the shaded area represents the proportion mis-classified. Thai $\mathrm{RC}=$ Thai Red Cross, Aus RC = Australian Red Cross, VBDR = Volunteer Blood Donor Registry. 
Table 1. Epidemiological overview of data sets. Individuals in the antigen discovery phase were recruited following a clinical episode of $P$. vivax. *denotes PCR prevalence in the general population.

\begin{tabular}{|c|c|c|c|c|c|c|c|c|c|}
\hline \multirow[t]{2}{*}{ population } & \multirow{2}{*}{$\begin{array}{l}\text { N: number of } \\
\text { participants }\end{array}$} & \multirow{2}{*}{$\begin{array}{l}\text { n: subset with } \\
\text { antibody } \\
\text { measurements }\end{array}$} & \multirow{2}{*}{$\begin{array}{l}\text { age (years) } \\
\text { median (range) }\end{array}$} & \multirow{2}{*}{$\begin{array}{l}\text { sex } \\
\% \text { female }\end{array}$} & \multirow[t]{2}{*}{ PCR PvPR } & \multicolumn{4}{|c|}{ time since last PCR positive sample } \\
\hline & & & & & & current & $<9$ months & 9-12 months & no detected \\
\hline \multicolumn{10}{|l|}{ (A) Antigen discovery data } \\
\hline Thailand & 57 & 32 & $29(7,71)$ & $44 \%$ & $11.4 \% *$ & - & - & - & - \\
\hline Brazil & 91 & 33 & $36(16,56)$ & $21 \%$ & $5.0 \% *$ & - & - & - & - \\
\hline \multicolumn{10}{|l|}{ (B) Validation data } \\
\hline Thailand & 999 & 829 & $24(1,78)$ & $54.7 \%$ & $3.33 \%$ & 25 & 47 & 25 & 732 \\
\hline Brazil & 1274 & 928 & $25(0,103)$ & $50.7 \%$ & $5.21 \%$ & 40 & 165 & 31 & 692 \\
\hline Solomon Islands & 860 & 754 & $5.6(0.5,12.7)$ & $51.6 \%$ & $11.92 \%$ & 93 & 172 & 29 & 566 \\
\hline Victorian Blood Donor Registry (VBDR) & 102 & 102 & $39(18,68)$ & $69.6 \%$ & $0.0 \%$ & 0 & 0 & 0 & 102 \\
\hline Australian Red Cross (ARC) & 100 & 100 & $52(18,77)$ & $54.0 \%$ & $0.0 \%$ & 0 & 0 & 0 & 100 \\
\hline Thai Red Cross (TRC) & 72 & 72 & - & - & $0.0 \%$ & 0 & 0 & 0 & 72 \\
\hline
\end{tabular}


Table 2. Purified $P$. vivax proteins used in the validation phase and their individual performance. Area under the curve (AUC) from the single antigen classifier is shown; proteins are listed in order of best individual performance. 8 of 60 proteins are shown here; see Table S2 for the extended and complete Table.

References listed are for the protein production and purification method.

\begin{tabular}{|c|c|c|c|c|c|c|}
\hline Protein ID $^{\mathrm{a}}$ & Gene Annotation $^{\mathrm{a}}$ & $\begin{array}{l}\text { Protein } \\
\text { length, aa }\end{array}$ & $\begin{array}{l}\text { Construct, aa } \\
\text { (size) }\end{array}$ & Expression System $^{\mathrm{c}}$ & Purification Method & AUC \\
\hline PVX_094255B & reticulocyte binding protein $2 \mathrm{~b}$ (RBP2b) & 2806 & $161-1454(1294)$ & E. coli & $\begin{array}{l}2 x \text { affinity }+ \text { size exclusion }(27,30 \text {, } \\
57)\end{array}$ & 0.819 \\
\hline PVX_099980 & merozoite surface protein 1 (MSP1-19) & 1751 & $1622-1729(108)$ & WGCF & One-step Ni column & 0.804 \\
\hline PVX_000930 & sexual stage antigen s16, putative & 140 & 31-end (110) & WGCF & One-step Ni column & 0.78 \\
\hline PVX_094255A & reticulocyte binding protein $2 b(\mathrm{RBP} 2 \mathrm{~b})$ & 2806 & $1986-2653(667)$ & WGCF & One-step Ni column & 0.779 \\
\hline PVX_087885B & rhoptry-associated membrane antigen, putative & 730 & $462-730(269)$ & WGCF & One-step Ni column $(28)$ & 0.774 \\
\hline PVX_095055 & Rh5 interacting protein, putative (RIPR) & 1075 & $552-1075(524)$ & E. coli & $2 \mathrm{x}$ affinity + size exclusion $(59)$ & 0.767 \\
\hline AAY34130.1 ${ }^{\mathrm{b}}$ & Duffy binding protein (DBP, region $2, \mathrm{AH}$ strain) & 237 & $1-237(237)$ & E. coli & $\mathrm{Ni}$, ion exchange, gel filtration (58) & 0.764 \\
\hline PVX_097715 & hypothetical protein & 450 & 20-end (431) & WGCF & One-step Ni column & 0.76 \\
\hline
\end{tabular}

${ }^{\mathrm{a}}$ PlasmoDB release 36 (http://plasmodb.org/plasmo/), ${ }^{\mathrm{b}} \mathrm{GenBank}$ 